\title{
VASCULAR PLANT BIODIVERSITY OF FLOODPLAIN FOREST GEOBIOCOENOSIS IN LOWER MORAVA RIVER BASIN (FOREST DISTRICT TVRDONICE), CZECH REPUBLIC
}

\author{
PETR MADĚRA, RADOMÍr ŘEPKA, JAN ŠEBESTA, TOMÁŠ KOUTECKÝ, \\ MARTIN KLIMÁNEK
}

Faculty of Forestry and Wood Technology Mendel University in Brno, Zemědělská 3, 613 00, Brno, Czech Republic, e-mail: petrmad@mendelu.cz

Received: $9^{\text {th }}$ September 2013, Accepted: $3^{\text {rd }}$ November 2013

\begin{abstract}
This paper presents an evaluation of full-area floristic mapping of floodplain forest in Tvrdonice forest district (Židlochovice Forest Enterprise) based on a single forest stand inventory. The study area encompasses 2,200 ha of forests, where 769 segments were inventoried, and 46,886 single records about presence of vascular plant species were catalogued. We found 612 species (incl. subspecies and hybrids), out of which 514 were herbs, 98 were woody plants, 113 were endangered species and 170 were adventive species. The average area of a segment is 2.86 ha. The mean number of species per segment is 60.97 in a range of 4-151.
\end{abstract}

Key words: biodiversity, vascular plants, floodplain forest, forest district Tvrdonice, Czech Republic

\section{INTRODUCTION}

Formations of floodplain forests in Europe are classified as azonal; however, their vegetation differs in particular parts of Europe both in its physiognomy and species composition (Bohn et al. 2003). Floodplains and floodplain forests in alluvia of large rivers are dynamic ecosystems, which are subject to fast changes in the temporal as well as spatial sense (Klimo et al. 2008). They are relatively young communities, as regards their development, and are affected by two main ecological factors - more or less cyclic flooding and a high level of the groundwater (Madera et al. 2008). Their genesis and especially florogenesis has also been affected by the broad surroundings of the floodplains, i.e. the entire river drainage basin. The cyclic character of floods and the migration of diaspores of many species by water (Boedeltje 2004) were the factors that have been enriching the flora of floodplain forests for centuries. Considering the ever more frequent deposition of material from the entire drainage basins and global eutrophication on the one hand, as well as the natural fast decomposition of organic mass on the other hand, these sites are very well supplied with nutrients and in recent decades have also been supplied or even oversaturated with nitrogen. The significance of floodplain forests for biodiversity has been mentioned in many studies (Tabacchi et al. 1996, Naiman \& Decamps 1997, 
Schnitzler et al. 2007); however, the condition of river ecosystems in Europe is currently affected by a number of negative anthropogenic factors (Wenger et al. 1990).

The biodiversity of vascular plants in the study area has been affected significantly by the forest management in the area, i.e. the way the landscape in the entire drainage basin has been managed. The floodplain was densely populated in the period of Great Moravia. In the Middle Ages, the forest communities were harvested with very short rotation periods (as frequently as 7 years), managed as coppice forests or coppice with standards. Pasture was created in the floodplain forests in the historical times and the forest was regenerated by agroforestry (Nožička 1956; Hrib 2004) and in the most recent decades by various forms of uniform clear-cutting with broadcast soil preparation. Regeneration has always been achieved using autochthonous woody plants; therefore, nowadays, there are valuable forest stands that can be referred to as man-made natural ecosystems (Maarel 1975).

South-Moravian lowland floodplains, together with the adjacent Slovakian and Lower Austrian floodplains, are the most extensive complex within central Europe; recently, they have been a subject of research of more and more studies. They are significant forest coenoses covering the broad floodplains of lower reaches of large rivers, well-known for their dynamic development (Maděra 2001a, 2001b) and high production of biomass (Klimo \& Hager 2000; Penka et al. 1985). The afore-mentioned floodplain forests in the Czech Republic are managed by the Židlochovice Forest Enterprise and are divided into three forest districts (Valtice, Tvrdonice and Soutok). Floristic-oriented studies from the area of the confluence of the Morava and the Dyje rivers have been published only recently. Horák (1961) focused on the typology of floodplain forests, Vicherek et al. (2000) dealt with a floristic inventory in map squares regardless of forest or non-forest biotopes, Danihelka et al. (1995) and Danihelka \& Šumberová (2004) described the distribution of selected taxa. Maděra et al. (2011) presented the results of a floristic inventory of floodplain forests within the Valtice forest district.

The aims of this paper are to continue the work presented in the last mentioned publication and introduce another stage of the floristic inventory of floodplain forests, this time within the Tvrdonice forest district of the Židlochovice Forest Enterprise, as a model of diversity of vascular plants in floodplain forests of the Lower Morava Basin. The results should serve as a significant source of information for the zonation of Dolní Morava Biosphere Reserve, the studied area being its part.

\section{MATERIAL AND METHODS}

\section{Study area}

The area of 2,200 ha of floodplain forest along the lower reaches of the Morava river between towns Lanžhot and Hodonín was inventoried. This is the Tvrdonice forest district, Židlochovice Forest Enterprise, which is a part of state forests managed by Lesy ČR s.p. The Morava river in the studied area forms the border between the Czech Republic and Slovakia (Fig.1). A short description of the historical development of the area and its significance for nature conservation was presented in the previous study (Maděra et al. 2011), which concerned the Valtice forest district. 
Fig. 1: Study area

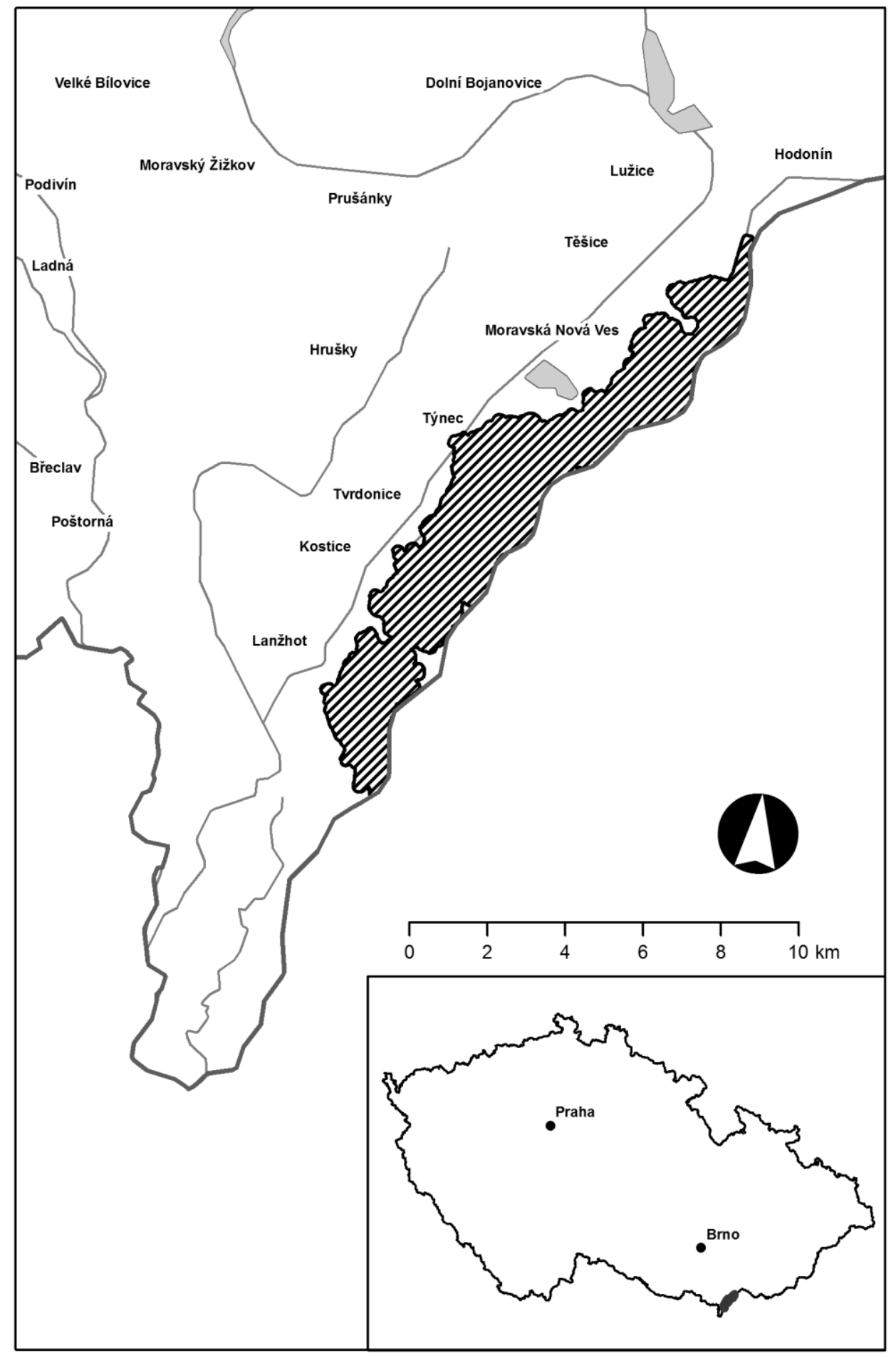




\section{Methods}

All vascular plants in the area of the Tvrdonice forest district were recorded between 2007 and 2011 down to the level of a segment; each segment corresponds to one stand group (exceptionally, similar groups are put together or non-homogeneous groups are divided). The presence of species in each segment is ticked in a list that includes 263 most common species of herbs in south-Moravian floodplains. Rare species and woody plants are added to the list. We used nomenclature according to Kubát et al. (2002). The occurrence of species growing only at the segment edges (stand adjacent to a forest road, a water current, a clearing, a meadow) and dominant species (species of over $40 \%$ cover) are marked differently. The terrain survey needs to be conducted in two aspects: spring (March 20May 31) and summer (June 1-November 30); also fresh clearings and young plantings were inventoried. The ticking lists are then transferred to a database and further processed. The segment after digitalisation becomes a site (a point in the point map). The digitalisation and creation of the species distribution maps was implemented in the GIS environment (ArcGIS).

\section{RESULTS}

The total study area is 2,200 ha of forest; 769 segments were explored and 46,886 records on the presence of vascular plant taxa were taken. According to the records, there are 612 species (and lower taxa, or hybrids) in the area, out of which there are 514 herbs and 98 woody plants. The mean size of a segment is 2.86 ha. On average, there are 60.97 taxa (range of 4-151) per segment (most segments containing 40-59 species). The numbers of species within a segment are distributed slightly unequally - there are more segments with lower numbers of species than average (412) and fewer segments with higher numbers (357) (Fig.2). On average, there are 9.39 species of woody plants and 51.58 species of herbs in a segment. The spatial distribution of the segments with their highlighted significance for biodiversity (the number of species per segment) is illustrated in Fig. 3.

Fig. 2: Frequency of segments according to containing number of species

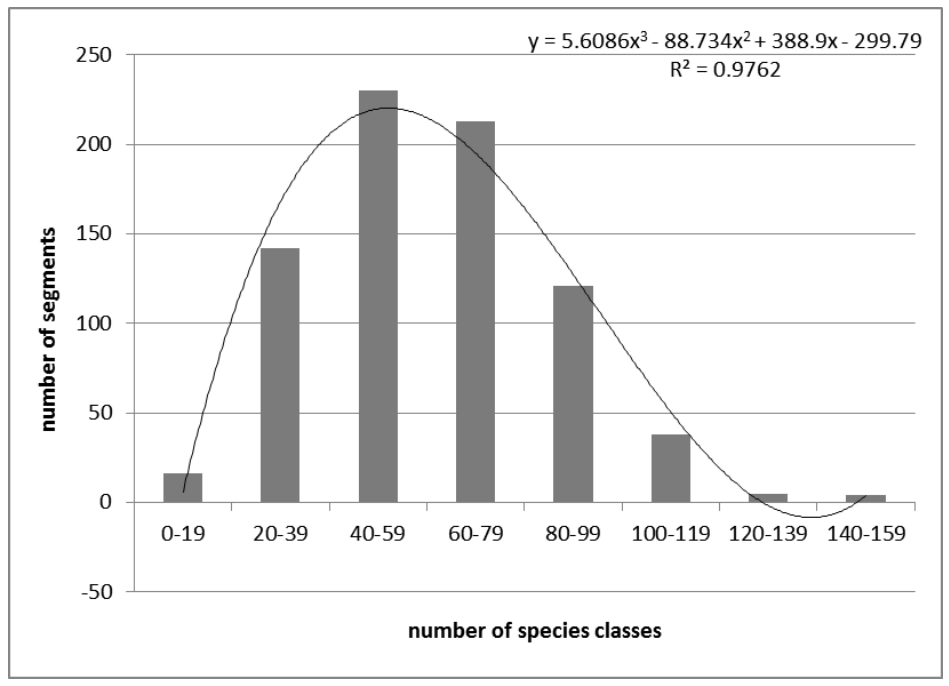


Fig. 3: Map of the number of all vascular plant species per segment
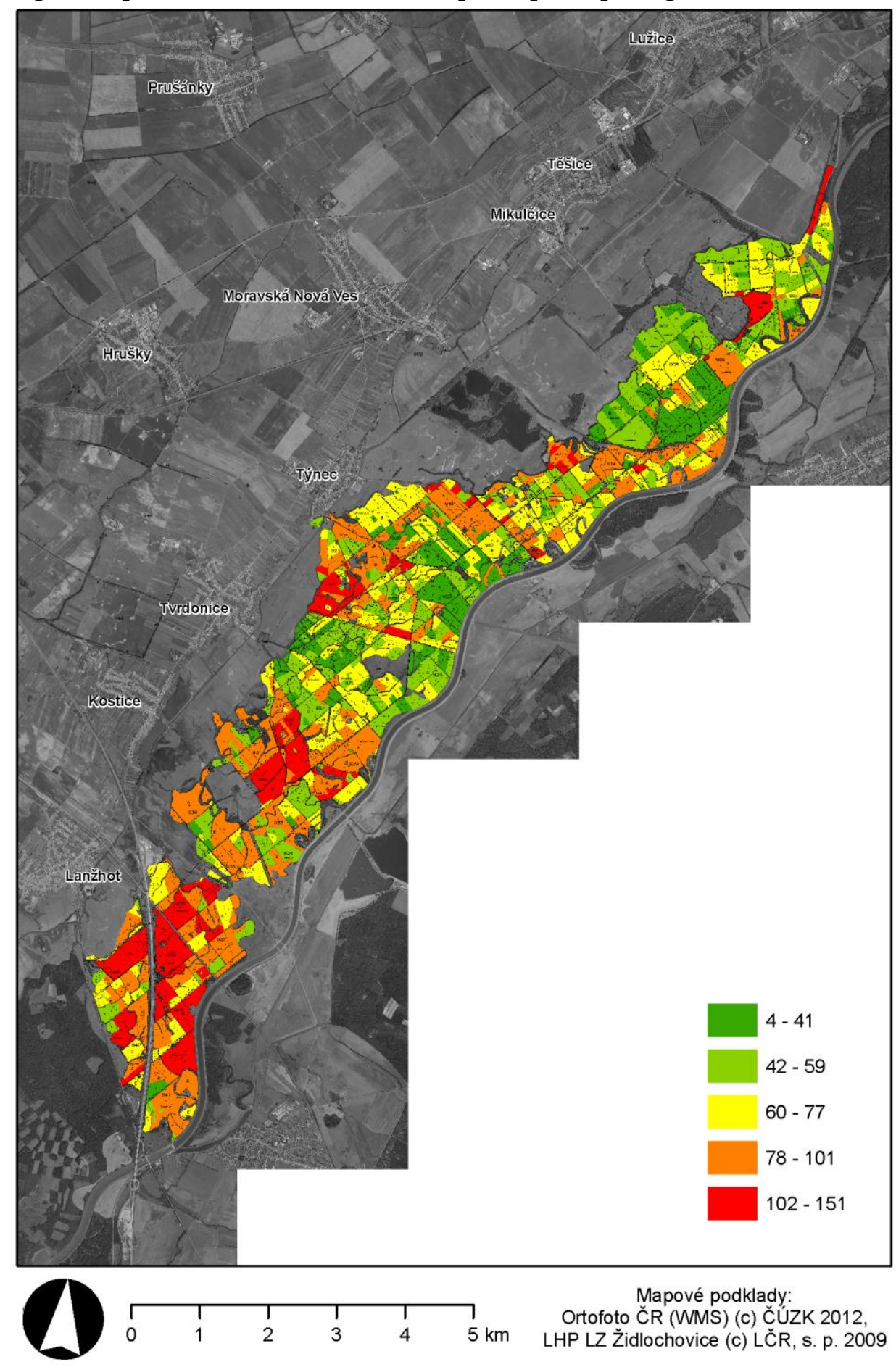

Mapové podklady:

Ortofoto ČR (WMS) (c) ČÚZK 2012,

LHP LZ Židlochovice (c) LČR, s. p. 2009 
We also examined the frequency of species occurrence (presence of a taxon in segments) in the area (Table 1). The analysis shows that 110 species (i.e. nearly $15 \%$ ) occur in one segment only, 281 species (i.e. 37\%) are present in 1-9 segments; it means that the species scarcely occur in the area and are rare. The table also shows that another 196 species (i.e. $25 \%$ ) are present in 10-99 segments. These species can be referred to as scattered. 135 species (i.e. 17.5.\%) are present in over 100 segments - these species are abundant. Only 25 species occur in over $60 \%$ of segments (Table 2) - the species with high stability. This group contains most species of the herb layer of floodplain forests considered typical species of subclass Ulmenion (Neuhäuslová 2001). Only two adventive species are in this group - invasive neophyte Aster lanceolatus and naturalised archeophyte Arctium lappa.

Table 1: The frequency of species occurrence

\begin{tabular}{|l|c|}
\hline $\begin{array}{c}\text { Classes of } \\
\text { segments number }\end{array}$ & $\begin{array}{c}\text { Number of } \\
\text { species }\end{array}$ \\
\hline $700+$ & 2 \\
\hline $600-699$ & 8 \\
\hline $500-599$ & 12 \\
\hline $400-499$ & 13 \\
\hline $300-399$ & 17 \\
\hline $200-299$ & 26 \\
\hline $100-199$ & 57 \\
\hline $0-99$ & 477 \\
\hline $90-99$ & 0 \\
\hline $80-89$ & 9 \\
\hline $70-79$ & 14 \\
\hline $60-69$ & 17 \\
\hline $50-59$ & 16 \\
\hline $40-49$ & 14 \\
\hline $30-39$ & 23 \\
\hline $20-29$ & 36 \\
\hline $10-19$ & 67 \\
\hline $1-9$ & 281 \\
\hline
\end{tabular}


Table 2: Species with occurence frequency over $60 \%$ of segments

\begin{tabular}{|l|c|}
\hline \multicolumn{1}{|c|}{ Species } & No. of segments \\
\hline Aster lanceolatus & 731 \\
\hline Rubus caesius & 708 \\
\hline Urtica dioica & 694 \\
\hline Geum urbanum & 670 \\
\hline Glechoma hederacea & 653 \\
\hline Carex riparia & 626 \\
\hline Galium aparine & 626 \\
\hline Quercus robur & 624 \\
\hline Acer campestre & 608 \\
\hline Symphytum officinale & 603 \\
\hline Brachypodium sylvaticum & 599 \\
\hline Festuca gigantea & 594 \\
\hline Rumex sanguineus & 594 \\
\hline Arctium lappa & 573 \\
\hline Deschampsia cespitosa & 569 \\
\hline Torilis japonica & 565 \\
\hline Phalaris arundinacea & 555 \\
\hline Iris pseudacorus & 539 \\
\hline Lysimachia nummularia & 534 \\
\hline Fraxinus angustifolia & 512 \\
\hline Cirsium arvense & 512 \\
\hline Dactylis polygama & 509 \\
\hline Taraxacum sect. Ruderalia & 493 \\
\hline Ficaria verna & 481 \\
\hline Poa palustris & 471 \\
\hline
\end{tabular}

From the perspective of nature preservation, it is interesting to evaluate the proportion of adventive species (based on Pyšek et al. 2002) and endangered species (based on Holub \& Procházka 2000). Considering merely the number of species (Fig. 4), over a quarter (27.7\%, i.e. 170 taxa) are various categories of adventive species and $18.4 \%$ (113) taxa are species with various levels of conservation status. However, Fig. 5 has a higher information capacity concerning the role of these groups in the area. It shows the results categorised based on the number of records of the species in the segments. Based on this, the proportion of adventive species drops to $18 \%$ (8,455 records) and the proportion of endangered species to $7 \%$ (3,478 records). Neither of this is sufficiently informative, as the presence of taxa in segments does not give any idea about their cover. For example, Aster lanceolatus, a significant invasive neophyte, is present in over $95 \%$ of segments and it is dominant in the 
herb layer synusia in $18 \%$ of them; the dominance is the most frequent in stands up to 10 years of age (Řepka \& Maděra 2009a). By contrast, Arctium lappa is present in $74 \%$ of segments, but always relatively scarcely.

Fig. 4: Proportion of adventive, threatened and others vascular plant species in the area of study

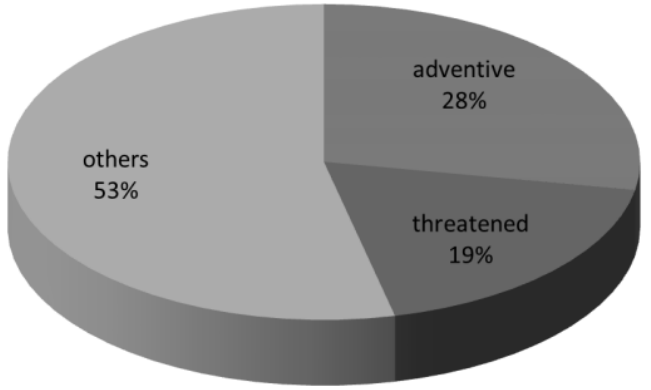

Fig. 5: Proportion of adventive, threatened and others vascular plant species in the area of study according to the number of records

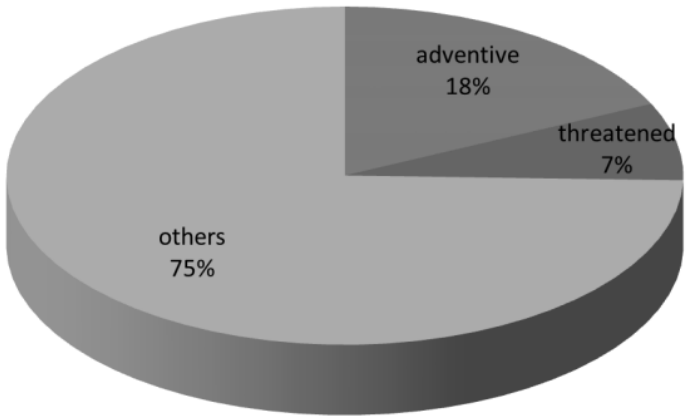

Within the set of adventive species, archeophytes (58.8\%) slightly prevail over neophytes $(41,2 \%)$; there are 48 , i.e. $28 \%$ of invasive species in total (Fig. 6, Table 3), according to Pyšek et al. (2002) we distinguish archeophytes (arc) and neophytes (neo), in detail (cas casual, nat - naturalised and inv - invasive). On average, there are 11 adventive species in a segment (range of 0-46). Only 3 segments contained no adventive species. There were up to $10 \%$ of adventive species in 124 segments, $10-20 \%$ in 403 segments, $20-30 \%$ in 198 segments, $31-40 \%$ in 34 segments, and $41-50 \%$ of adventive species in 8 segments. Two segments even contains over $50 \%$ of adventive species. The loading of individual segments by the presence of adventive species is illustrated in the map, Fig. 7, neophytes especially are pictured in the map, Fig. 8. 
Table 3: The abundance of different categories of adventive species (according to Pyšek et al. 2002)

\begin{tabular}{|c|c|c|c|c|c|c|}
\hline \multirow{2}{*}{$\begin{array}{c}\text { adventive } \\
\text { species } \\
\text { category }\end{array}$} & \multicolumn{2}{|c|}{ all species } & \multicolumn{2}{c|}{ herbs } & \multicolumn{2}{c|}{ woody plants } \\
\cline { 2 - 7 } & numbecies & $\begin{array}{c}\text { records } \\
\text { number }\end{array}$ & $\begin{array}{c}\text { species } \\
\text { number }\end{array}$ & $\begin{array}{c}\text { records } \\
\text { number }\end{array}$ & $\begin{array}{c}\text { species } \\
\text { number }\end{array}$ & $\begin{array}{c}\text { records } \\
\text { number }\end{array}$ \\
\hline ar cas & 8 & 90 & 5 & 8 & 3 & 82 \\
\hline ar nat & 77 & 3509 & 71 & 3443 & 6 & 66 \\
\hline ar inv & 15 & 1665 & 15 & 1665 & 0 & 0 \\
\hline neo cas & 17 & 142 & 5 & 11 & 12 & 131 \\
\hline neo nat & 20 & 626 & 16 & 590 & 4 & 36 \\
\hline neo inv & 33 & 2423 & 25 & 2043 & 8 & 380 \\
\hline
\end{tabular}

Fig. 6: Proportion of adventive species (according to Pyšek et al. 2002) in the area of study

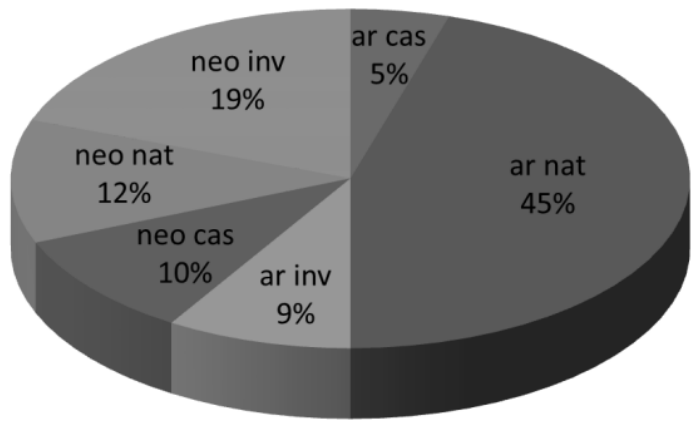


Fig. 7: Map of the number of adventive vascular plant species per segment
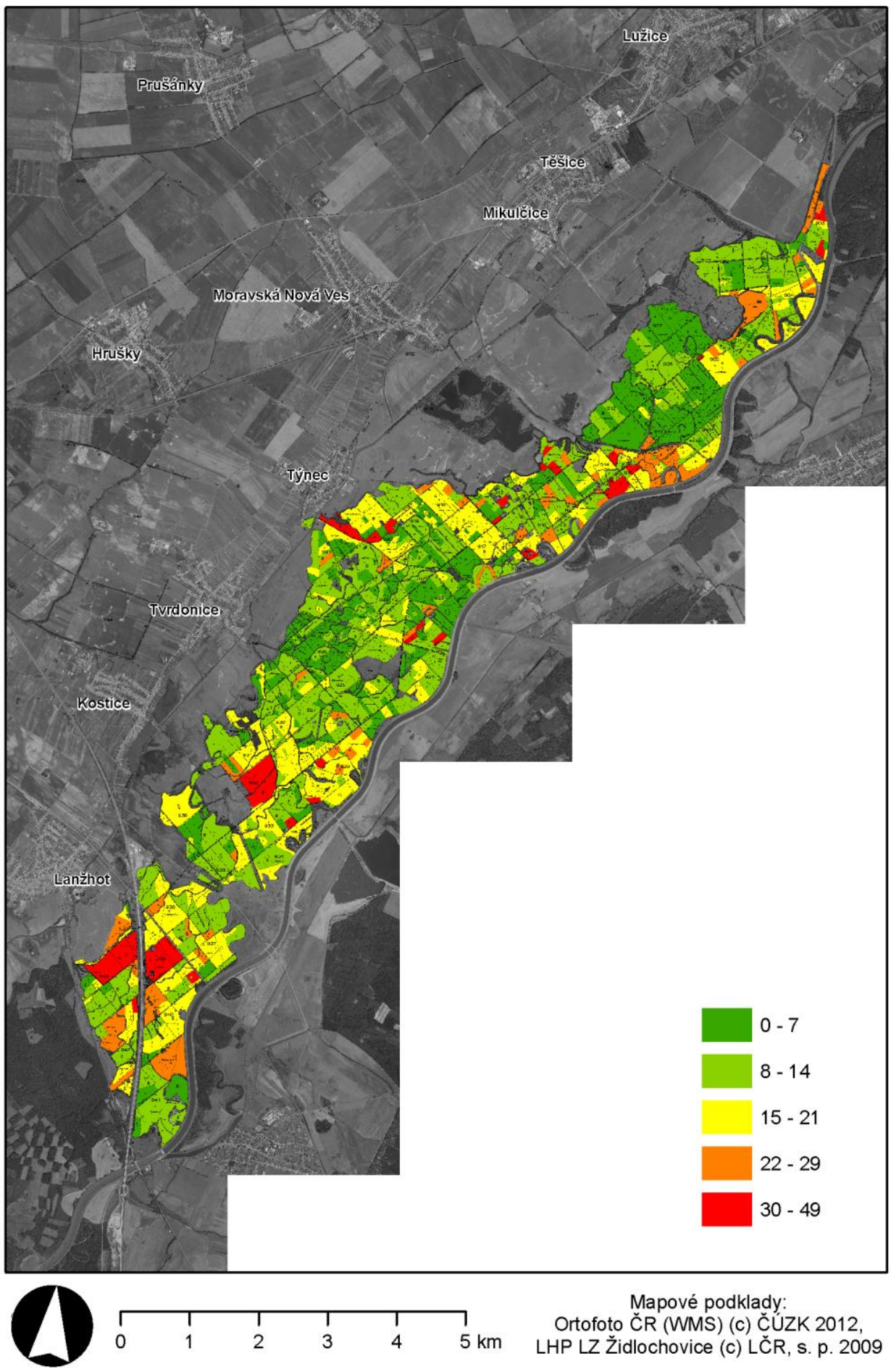
Fig. 8: Map of the number of vascular plant neophytes per segment
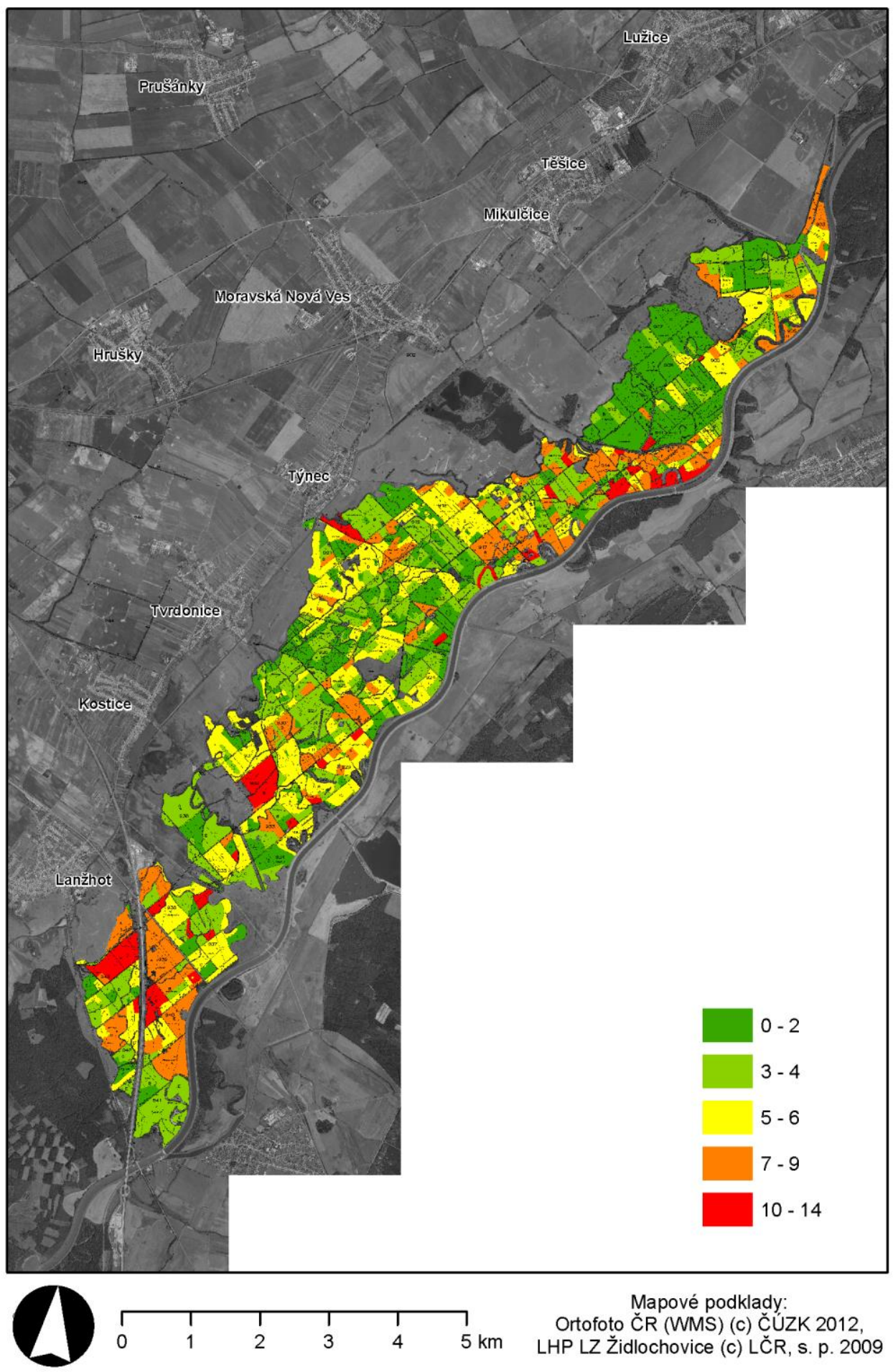

Mapové podklady:

Ortofoto ČR (WMS) (c) ČÚZK 2012, LHP LZ Židlochovice (c) LČR, s. p. 2009 
As concerns threatened species, $19.46 \%$ of species are protected by law, the rest are within various categories of the Red List. There are 8 critically endangered species, 34 strongly endangered and 25 endangered, the other 46 species are within C4 category requiring further attention (Fig. 9, Table 4). The analysis shows that the mean number per segment is 4.52 species (range of $0-17$ ). Threatened species are not present in 14 segments only; in the others there is at least one threatened species. Most segments (307) contain 6$9 \%$ of threatened species; 10 segments even over $15 \%$. The most of threatened species $(66 \%)$ are present in 1-10 segments and only $7 \%$ of threatened species are present in over 100 segments. The spatial distribution of the numbers of threatened species of plants in the segments is shown in the map, Fig. 10. The map in Fig. 11 shows the species of categories C1 (critically endangered) and C2 (strongly endangered).

Table 4: The abundance of threatened vascular plants species (according to Holub \& Procházka 2000)

\begin{tabular}{|c|c|c|c|c|c|c|}
\hline \multirow{2}{*}{$\begin{array}{c}\text { threat and } \\
\text { protection } \\
\text { category }\end{array}$} & \multicolumn{2}{|c|}{ all species } & \multicolumn{2}{c|}{ herbs } & \multicolumn{2}{c|}{ woody plants } \\
\cline { 2 - 7 } & numberies & $\begin{array}{c}\text { records } \\
\text { number }\end{array}$ & $\begin{array}{c}\text { species } \\
\text { number }\end{array}$ & $\begin{array}{c}\text { records } \\
\text { number }\end{array}$ & $\begin{array}{c}\text { species } \\
\text { number }\end{array}$ & $\begin{array}{c}\text { records } \\
\text { number }\end{array}$ \\
\hline$\S 1$ & 7 & 49 & 7 & 49 & 0 & 0 \\
\hline$\S 2$ & 11 & 72 & 11 & 72 & 0 & 0 \\
\hline$\S 3$ & 4 & 17 & 3 & 16 & 1 & 1 \\
\hline C1 & 8 & 62 & 8 & 62 & 0 & 0 \\
\hline C2 & 34 & 825 & 31 & 705 & 3 & 120 \\
\hline C3 & 25 & 749 & 23 & 236 & 2 & 513 \\
\hline C4 & 46 & 1842 & 36 & 1262 & 10 & 580 \\
\hline
\end{tabular}

Fig. 9: Proportion of threatened species in the area of study

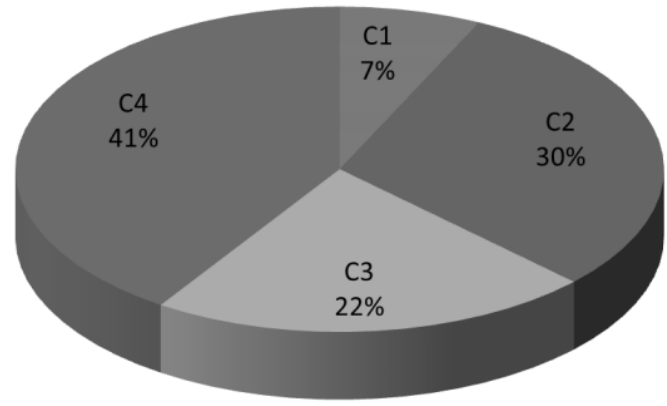


Fig. 10: Map of the number of endangered vascular plant species per segment according to Pyšek et al. (2002)
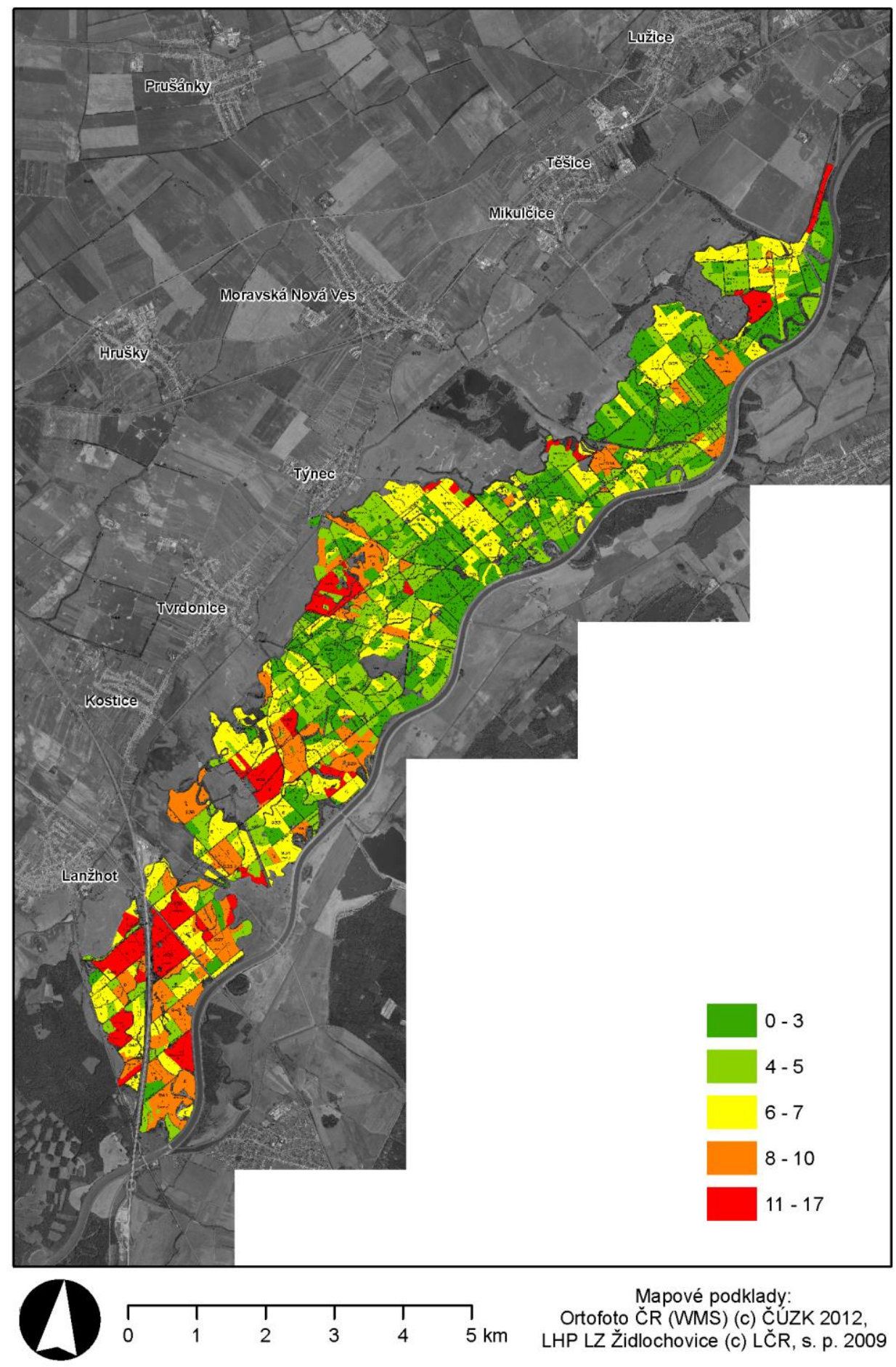

Mapové podklady:

Ortofoto ČR (WMS) (c) ČÚZK 2012,

LHP LZ Židlochovice (c) LČR, s. p. 2009 
Fig. 11: Map of the number of critical (C1) and strong endangered vascular (C2) plant species per segment according to Holub \& Procházka (2000)
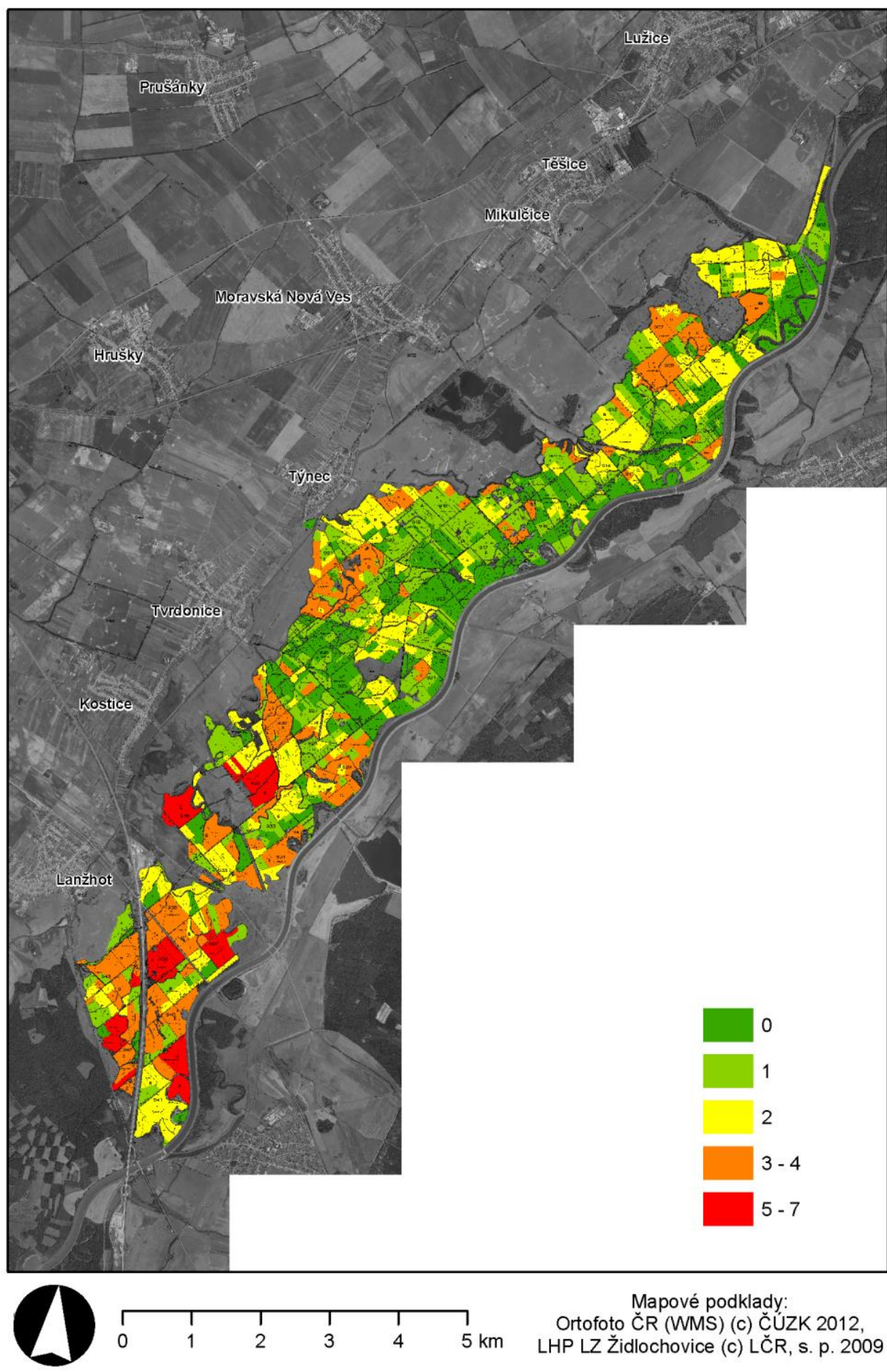

Mapové podklady:

Ortofoto ČR (WMS) (c) ČÚZK 2012,

LHP LZ Židlochovice (c) LČR, s. p. 2009 


\section{Diversity of woody plants in the floodplain forests}

As has been mentioned, we found 98 species, subspecies and hybrids of woody plants in the study area. Based on Úradníček et al. (2010), woody plants are not only trees and shrubs but also semi-shrubs (e.g. Solanum dulcamara) or woody lianas (e.g. Hedera helix) and shrublets, whose representative has not been found in the area (Fig.12).

\section{Fig. 12: Proportion of life form occurence of woody plants}

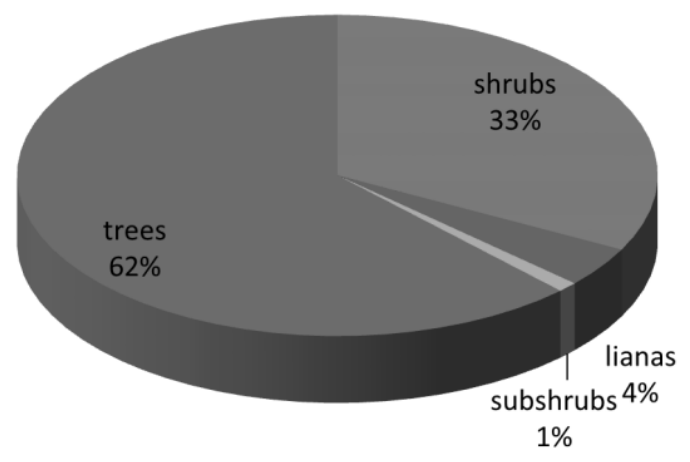

Out of the total number of woody plants found, there are 25 abundant species (occurrence in over 100 segments), 31 scattered species (10-99 segments) and 42 rare species (1-9 segments) - 23 species were recorded in one segment only.

From the perspective of autochthonous character, 33 recorded species are various types of adventive species (Table 5). There are 8 recorded invasive neophytes, a more significant presence being recorded for Acer negundo - in nearly $24 \%$ of segments and Populus $x$ canadensis - in $18 \%$ of segments. The former is the only one that propagates spontaneously, the others are grown (besides hybrid poplars, Fraxinus pennsylvanica and Quercus rubra) and spread less. Pyšek et al. (2002) also categorised the frequently grown Juglans nigra as an occasionally wild-growing neophyte; however, in the conditions of a floodplain we can assume at least a very good naturalisation as it often regenerates naturally - it was recorded in $10 \%$ of segments. 
Table 5: The presence of adventive woody plants species in segments (according to Pyšek et al. 2002)

\begin{tabular}{|c|c|c|c|c|}
\hline \multirow{2}{*}{\begin{tabular}{|l} 
Species \\
Malus domestica \\
\end{tabular}} & \multirow{2}{*}{$\begin{array}{c}\text { number of } \\
\text { segments }\end{array}$} & \multirow{2}{*}{$\begin{array}{c}\begin{array}{c}\text { proportion } \\
\text { of segments }\end{array} \\
9.49 \\
\end{array}$} & \multicolumn{2}{|c|}{$\begin{array}{c}\text { adventive species } \\
\text { category }\end{array}$} \\
\hline & & & cas & ar \\
\hline Morus alba & 5 & 0.65 & cas & ar \\
\hline Vitis vinifera subsp. vinifera & 4 & 0.52 & cas & ar \\
\hline Pyrus communis & 34 & 4.42 & nat & ar \\
\hline Malus $\mathrm{x}$ dasyphylla & 13 & 1.69 & nat & ar \\
\hline Prunus insititia & 11 & 1.43 & nat & ar \\
\hline Juglans regia & 5 & 0.65 & nat & ar \\
\hline Prunus domestica & 2 & 0.26 & nat & ar \\
\hline Prunus cerasus & 1 & 0.13 & nat & ar \\
\hline Juglans nigra & 78 & 10.14 & cas & neo \\
\hline Aesculus hippocastanum & 27 & 3.51 & cas & neo \\
\hline Tilia tomentosa & 15 & 1.95 & cas & neo \\
\hline Populus balsamifera & 2 & 0.26 & cas & neo \\
\hline Juglans $x$ intermedia & 2 & 0.26 & cas & neo \\
\hline Abies grandis & 1 & 0.13 & cas & neo \\
\hline Eleagnus angustifolia & 1 & 0.13 & cas & neo \\
\hline Gleditsia triacanthos & 1 & 0.13 & cas & neo \\
\hline Picea pungens & 1 & 0.13 & cas & neo \\
\hline Pinus jeffreyi & 1 & 0.13 & cas & neo \\
\hline Platanus $\mathrm{x}$ hispanica & 1 & 0.13 & cas & neo \\
\hline Thuja occidentalis & 1 & 0.13 & cas & neo \\
\hline Acer negundo & 184 & 23.93 & inv & neo \\
\hline Populus $\mathrm{x}$ canadensis & 139 & 18.08 & inv & neo \\
\hline Robinia pseudacacia & 25 & 3.25 & inv & neo \\
\hline Quercus rubra & 16 & 2.08 & inv & neo \\
\hline Fraxinus pennsylvanica & 12 & 1.56 & inv & neo \\
\hline Parthenocissus inserta & 4 & 0.52 & inv & neo \\
\hline Ailanthus altissima & 2 & 0.26 & inv & neo \\
\hline Mahonia aquifolium & 1 & 0.13 & inv & neo \\
\hline Physocarpus opulifolius & 1 & 0.13 & inv & neo \\
\hline Prunus cerasifera & 20 & 2.60 & nat & neo \\
\hline Ribes rubrum & 11 & 1.43 & nat & neo \\
\hline Pinus nigra & 1 & 0.13 & nat & neo \\
\hline
\end{tabular}


15 species of the woody plants fall within threatened species of some category (Table 6) but only Cornus mas is protected by law and it was found in one segment only. Floodplain forests are indispensable biotopes of strongly endangered woody species, Malus sylvestris (70 segments), Populus nigra (33 segments) and endangered Fraxinus angustifolia (512 segments). There is also a strong population of elms, both Ulmus laevis and Ulmus minor (348 and 151 segments, respectively).

Table 6: The presence of threatened woody plant species

\begin{tabular}{|l|c|c|}
\hline \multicolumn{1}{|c|}{ Species } & $\begin{array}{c}\text { threat } \\
\text { category }\end{array}$ & $\begin{array}{c}\text { number of } \\
\text { segments }\end{array}$ \\
\hline Cornus mas & $\S 3(\mathrm{C} 4)$ & 1 \\
\hline Malus sylvestris & $\mathrm{C} 2$ & 70 \\
\hline Populus nigra & $\mathrm{C} 2$ & 33 \\
\hline Quercus cerris & $\mathrm{C} 2$ & 17 \\
\hline Fraxinus angustifolia & $\mathrm{C} 3$ & 512 \\
\hline Prunus mahaleb & $\mathrm{C} 3$ & 1 \\
\hline Ulmus laevis & $\mathrm{C} 4$ & 348 \\
\hline Ulmus minor & $\mathrm{C} 4$ & 151 \\
\hline Viscum album & $\mathrm{C} 4$ & 28 \\
\hline Loranthus europaeus & $\mathrm{C} 4$ & 25 \\
\hline Pyrus pyraster & $\mathrm{C} 4$ & 20 \\
\hline Euonymus verrucosa & $\mathrm{C} 4$ & 3 \\
\hline Viburnum lantana & $\mathrm{C} 4$ & 2 \\
\hline Berberis vulgaris & $\mathrm{C} 4$ & 1 \\
\hline Sorbus aria & $\mathrm{C} 4$ & 1 \\
\hline
\end{tabular}

It is typical of floodplain woody plants that they grow to vast dimensions within their species (Maderra et al. 2007). Huge specimens can be found both in the stands and the meadows, riparian stands and forest edges with a high density. Their ecological significance is great; they are biotopes for a number of specially protected insects and birds, they form the landscape character, and they often represent the last remnants of local populations. Therefore, they need to be devoted sufficient attention in management (Rychtecká \& Dreslerová 2009).

\section{Diversity of herbs in the floodplain forests}

We determined 514 species, subspecies and hybrids of herbs in the synusia of floodplain forest herb layer. Out of the total number of herbs found, there are 112 abundant species (occurrence in over 100 segments), 161 scattered species (10-99 segments) and 241 rare species within the area (1-9 segments) - 90 species were found in one segment only.

From the perspective of autochthonous character, 137 recorded species are various types of adventive species (Table 7), out of which there are 91 archeophytes and 46 neophytes, 40 invasive species. The more significant invasive archeophytes are Cirsium arvense in $66 \%$ 
of segments, Plantago major (40\% of segments), Tanacetum vulgare (36\% of segments), Cirsium vulgare (31\% of segments) and Tripleurospermum inodorum (24\% of segments). Only the first mentioned species is dominant in forest edges and openings. The most significant and highly aggressive invasive neophyte in the area is Aster lanceolatus, whose presence in nearly $95 \%$ of segments and frequent dominance in younger and older stands of the floodplain forest presents a problem with almost no solution any more (̌̌epka \& Maděra 2009a). The other abundant invasive neophytes in the area are Impatiens parviflora, Bidens frondosa, Erigeron annuus, Solidago gigantea, Conyza canadensis, Rudbeckia laciniata, Helianthus tuberosus, Impatiens glandulifera and species of genus Amaranthus. These species usually grow in clearings and newly established cultures and only the first two mentioned ones penetrate into forest communities.

Table 7: The presence of adventive herb species in segments

(according to Pyšek et al. 2002)

\begin{tabular}{|c|c|c|c|c|}
\hline Species & $\begin{array}{l}\text { number of } \\
\text { segments }\end{array}$ & $\begin{array}{l}\text { proportion } \\
\text { of segments }\end{array}$ & $\operatorname{adve}$ & \\
\hline Avena sativa & 3 & 0.39 & cas & ar \\
\hline Panicum miliaceum & 2 & 0.26 & cas & ar \\
\hline Brassica napus & 1 & 0.13 & cas & ar \\
\hline Cannabis sativa & 1 & 0.13 & cas & ar \\
\hline Hordeum distichon & 1 & 0.13 & cas & ar \\
\hline Cirsium arvense & 512 & 66.49 & inv & ar \\
\hline Plantago major & 308 & 40.00 & inv & ar \\
\hline Tanacetum vulgare & 284 & 36.88 & inv & ar \\
\hline Cirsium vulgare & 239 & 31.04 & inv & ar \\
\hline Tripleurospermum inodorum & 188 & 24.42 & inv & ar \\
\hline Ballota nigra & 51 & 6.62 & inv & ar \\
\hline Viola odorata & 35 & 4.55 & inv & ar \\
\hline Melilotus alba & 19 & 2.47 & inv & ar \\
\hline Melilotus officinalis & 13 & 1.69 & inv & ar \\
\hline Atriplex sagittata & 5 & 0.65 & inv & ar \\
\hline Chenopodium pedunculare & 5 & 0.65 & inv & ar \\
\hline Bryonia alba & 2 & 0.26 & inv & ar \\
\hline Conium maculatum & 2 & 0.26 & inv & ar \\
\hline Atriplex oblongifolia & 1 & 0.13 & inv & ar \\
\hline Cardaria draba & 1 & 0.13 & inv & ar \\
\hline Arctium lappa & 573 & 74.42 & nat & ar \\
\hline Carduus crispus & 429 & 55.71 & nat & ar \\
\hline Lapsana communis & 351 & 45.58 & nat & ar \\
\hline Bromus sterilis & 170 & 22.08 & nat & ar \\
\hline Echinochloa crus-galli & 139 & 18.05 & nat & ar \\
\hline Lactuca serriola & 132 & 17.14 & nat & ar \\
\hline Polygonum aviculare & 118 & 15.32 & nat & ar \\
\hline Chenopodium polyspermum & 102 & 13.25 & nat & ar \\
\hline Mentha arvensis & 89 & 11.56 & nat & ar \\
\hline Sonchus asper & 84 & 10.91 & nat & ar \\
\hline Atriplex patula & 79 & 10.26 & nat & ar \\
\hline Pastinaca sativa & 77 & 10.00 & nat & ar \\
\hline Chelidonium majus & 74 & 9.61 & nat & ar \\
\hline Capsella bursa-pastoris & 73 & 9.48 & nat & ar \\
\hline Sonchus arvensis & 67 & 8.70 & nat & ar \\
\hline Setaria pumila & 66 & 8.57 & nat & ar \\
\hline
\end{tabular}


Journal of Landscape Ecology (2013), Vol: 6 / No. 2.

\begin{tabular}{|c|c|c|c|c|}
\hline Species & $\begin{array}{l}\text { number of } \\
\text { segments }\end{array}$ & $\begin{array}{c}\text { proportion } \\
\text { of segments }\end{array}$ & adve & \\
\hline Medicago lupulina & 62 & 8.05 & nat & ar \\
\hline Lamium album & 58 & 7.53 & nat & ar \\
\hline Cichorium intybus & 57 & 7.40 & nat & ar \\
\hline Lamium purpureum & 50 & 6.49 & nat & ar \\
\hline Silene latifolia & 47 & 6.10 & nat & ar \\
\hline Fallopia convolvulus & 44 & 5.71 & nat & ar \\
\hline Bromus tectorum & 43 & 5.58 & nat & ar \\
\hline Vicia hirsuta & 42 & 5.45 & nat & ar \\
\hline Sonchus oleraceus & 36 & 4.68 & nat & ar \\
\hline Bromus hordeaceus & 31 & 4.03 & nat & ar \\
\hline Portulaca oleracea & 27 & 3.51 & nat & ar \\
\hline Solanum nigrum & 24 & 3.12 & nat & ar \\
\hline Convolvulus arvensis & 23 & 2.99 & nat & ar \\
\hline Carduus acanthoides & 22 & 2.86 & nat & ar \\
\hline Setaria viridis & 22 & 2.86 & nat & ar \\
\hline Crepis biennis & 20 & 2.60 & nat & ar \\
\hline Bromus commutatus & 19 & 2.47 & nat & ar \\
\hline Linaria vulgaris & 18 & 2.34 & nat & ar \\
\hline Vicia angustifolia & 17 & 2.21 & nat & ar \\
\hline Arctium tomentosum & 14 & 1.82 & nat & ar \\
\hline Sambucus ebulus & 12 & 1.56 & nat & ar \\
\hline Erysimum cheiranthoides & 10 & 1.30 & nat & ar \\
\hline Geranium pusillum & 10 & 1.30 & nat & ar \\
\hline Setaria verticillata & 10 & 1.30 & nat & ar \\
\hline Veronica arvensis & 10 & 1.30 & nat & ar \\
\hline Digitaria sanguinalis & 9 & 1.17 & nat & ar \\
\hline Senecio vulgaris & 9 & 1.17 & nat & ar \\
\hline Verbena officinalis & 9 & 1.17 & nat & ar \\
\hline Crepis capillaris & 7 & 0.91 & nat & ar \\
\hline Tragopogon dubius & 6 & 0.78 & nat & ar \\
\hline Leonurus cardiaca & 5 & 0.65 & nat & ar \\
\hline Armoracia rusticana & 4 & 0.52 & nat & ar \\
\hline Saponaria officinalis & 4 & 0.52 & nat & ar \\
\hline Bromus japonicus & 3 & 0.39 & nat & ar \\
\hline Eragrostis minor & 3 & 0.39 & nat & ar \\
\hline Hordeum murinum & 3 & 0.39 & nat & ar \\
\hline Myosotis arvensis & 3 & 0.39 & nat & ar \\
\hline Synapis arvensis & 3 & 0.39 & nat & ar \\
\hline Vicia villosa & 3 & 0.39 & nat & ar \\
\hline Descurainia sophia & 2 & 0.26 & nat & ar \\
\hline Lathyrus tuberosus & 2 & 0.26 & nat & ar \\
\hline Thlaspi arvense & 2 & 0.26 & nat & ar \\
\hline Vicia sativa & 2 & 0.26 & nat & ar \\
\hline Vicia villosa subsp. varia & 2 & 0.26 & nat & ar \\
\hline Arctium lappa $x$ tomentosum & 1 & 0.13 & nat & ar \\
\hline Avena fatua & 1 & 0.13 & nat & ar \\
\hline Berteroa incana & 1 & 0.13 & nat & ar \\
\hline Crepis tectorum & 1 & 0.13 & nat & ar \\
\hline Cynodon dactylon & 1 & 0.13 & nat & ar \\
\hline Euphorbia peplus & 1 & 0.13 & nat & ar \\
\hline Malva neglecta & 1 & 0.13 & nat & ar \\
\hline Nepeta cataria & 1 & 0.13 & nat & ar \\
\hline
\end{tabular}




\begin{tabular}{|c|c|c|c|c|}
\hline Species & $\begin{array}{l}\text { number of } \\
\text { segments }\end{array}$ & $\begin{array}{c}\text { proportion } \\
\text { of segments }\end{array}$ & & cies \\
\hline Papaver rhoeas & 1 & 0.13 & nat & ar \\
\hline Parietaria officinalis & 1 & 0.13 & nat & ar \\
\hline Physalis alkekengi & 1 & 0.13 & nat & ar \\
\hline Sagittaria latifolia & 6 & 0.78 & cas & neo \\
\hline Bromus carinatus & 2 & 0.26 & cas & neo \\
\hline Crepis foetida & 1 & 0.13 & cas & neo \\
\hline Helianthus annuus & 1 & 0.13 & cas & neo \\
\hline Zea mays & 1 & 0.13 & cas & neo \\
\hline Aster lanceolatus & 731 & 94.94 & inv & neo \\
\hline Impatiens parviflora & 274 & 35.58 & inv & neo \\
\hline Bidens frondosa & 267 & 34.68 & inv & neo \\
\hline Conyza canadensis & 174 & 22.60 & inv & neo \\
\hline Solidago gigantea & 168 & 21.82 & inv & neo \\
\hline Rudbeckia laciniata & 80 & 10.39 & inv & neo \\
\hline Helianthus tuberosus & 66 & 8.57 & inv & neo \\
\hline Impatiens glandulifera & 63 & 8.18 & inv & neo \\
\hline Arrhenatherum elatius & 60 & 7.79 & inv & neo \\
\hline Amaranthus retroflexus & 44 & 5.71 & inv & neo \\
\hline Amaranthus powellii & 30 & 3.90 & inv & neo \\
\hline Solidago canadensis & 27 & 3.51 & inv & neo \\
\hline Epilobium ciliatum & 22 & 2.86 & inv & neo \\
\hline Echinocystis lobata & 14 & 1.82 & inv & neo \\
\hline Reynoutria japonica & 6 & 0.78 & inv & neo \\
\hline Galinsoga parviflora & 3 & 0.39 & inv & neo \\
\hline Juncus tenuis & 3 & 0.39 & inv & neo \\
\hline Geranium pyrenaicum & 2 & 0.26 & inv & neo \\
\hline Matricaria discoidea & 2 & 0.26 & inv & neo \\
\hline Rumex thyrsiflorus & 2 & 0.26 & inv & neo \\
\hline Aster novi-belgii & 1 & 0.13 & inv & neo \\
\hline Galinsoga quadriradiata & 1 & 0.13 & inv & neo \\
\hline Oenothera biennis & 1 & 0.13 & inv & neo \\
\hline Reynoutria sachalinensis & 1 & 0.13 & inv & neo \\
\hline Veronica persica & 1 & 0.13 & inv & neo \\
\hline Erigeron annuus & 255 & 33.12 & nat & neo \\
\hline Oxalis fontana & 141 & 18.31 & nat & neo \\
\hline Trifolium hybridum & 129 & 16.75 & nat & neo \\
\hline Galega officinalis & 20 & 2.60 & nat & neo \\
\hline Chenopodium strictum & 14 & 1.82 & nat & neo \\
\hline Datura stramonium & 7 & 0.91 & nat & neo \\
\hline Xanthium albinum & 6 & 0.78 & nat & neo \\
\hline Agrostis gigantea & 4 & 0.52 & nat & neo \\
\hline Medicago sativa & 4 & 0.52 & nat & neo \\
\hline Amaranthus albus & 2 & 0.26 & nat & neo \\
\hline Asclepias syriaca & 2 & 0.26 & nat & neo \\
\hline Sisymbrium strictissimum & 2 & 0.26 & nat & neo \\
\hline Acorus calamus & 1 & 0.13 & nat & neo \\
\hline Erechtites hieraciifolia & 1 & 0.13 & nat & neo \\
\hline Oxalis corniculata & 1 & 0.13 & nat & neo \\
\hline Oxalis dillenii & 1 & 0.13 & nat & neo \\
\hline
\end{tabular}


As regards specially protected and threatened species, there are 98 of them in the area (Table 8). 21 species within the total number of 137 records in the segments are protected by law. Floodplain forests are very significant biotopes for species such as Leucojum aestivum (37 of segments), Euphorbia palustris (29), Carex strigosa (241), Carex divulsa (47), Cardamine dentata (123) and Carex riparia (626). Other species, indicating heavytextured soils with varying humidity or subhalophilous, occur mainly in floodplain meadows and edges or clearings within forests - Viola elatior, Scutellaria hastifolia, Carex melanostachya, Gratiola officinalis, Teucrium scordium, Lathyrus palustris, Pulicaria dysenterica, Leonurus marubiastrum, Lycopus exaltatus, Sonchus palustris, Lythrum virgatum, Sonchus palustris, Cnidium dubium, Silaum silaus, Trifolium fragiferum, Inula salicina, and Galium boreale. Marshlands, water streams and their edges are important biotopes for species such as Juncus atratus, Cardamine parviflora, Ceratophyllum submersum, Hottonia palustris, Sium latifolium, Potamogeton nodosus, Hydrocharis morsus-ranae, Najas marina, Butomus umbellatus, Cardamine matthioli, Leersia oryzoides, Scrophularia umbrosa or Veronica scutellata, Carex buekii. Elevated sandy dunes contain species such as Scilla drunensis, Galanthus nivalis, Equisetum ramosissimum.

Table 8: The presence of threatened herb species

\begin{tabular}{|l|c|c|}
\hline \multicolumn{1}{|c|}{ Species } & $\begin{array}{c}\text { threat } \\
\text { category }\end{array}$ & $\begin{array}{c}\text { number of } \\
\text { segments }\end{array}$ \\
\hline Leucojum aestivum & $\S 1, \mathrm{C} 1$ & 37 \\
\hline Cardamine parviflora & $\S 1, \mathrm{C} 1$ & 5 \\
\hline Viola elatior & $\S 1, \mathrm{C} 1$ & 3 \\
\hline Euphorbia lucida & $\S 1, \mathrm{C} 1$ & 1 \\
\hline Juncus atratus & $\S 1, \mathrm{C} 1$ & 1 \\
\hline Lathyrus palustris & $\S 1, \mathrm{C} 2$ & 1 \\
\hline Euphorbia palustris & $\S 2, \mathrm{C} 2$ & 29 \\
\hline Thalictrum flavum & $\S 2, \mathrm{C} 2$ & 11 \\
\hline Senecio sarracenicus & $\S 2, \mathrm{C} 2$ & 8 \\
\hline Scutellaria hastifolia & $\S 2, \mathrm{C} 2$ & 7 \\
\hline Gratiola officinalis & $\S 2, \mathrm{C} 2$ & 6 \\
\hline Allium angulosum & $\S 2, \mathrm{C} 2$ & 5 \\
\hline Carex melanostachya & $\S 2, \mathrm{C} 2$ & 2 \\
\hline Ceratophyllum submersum & $\S 2, \mathrm{C} 1$ & 1 \\
\hline Scilla drunensis & $\S 2, \mathrm{C} 2$ & 1 \\
\hline Teucrium scordium & $\S 2, \mathrm{C} 2$ & 1 \\
\hline Viola pumila & $\S 2, \mathrm{C} 2$ & 1 \\
\hline Epipactis albensis & $\S 2, \mathrm{C} 2$ & 1 \\
\hline Hottonia palustris & $\S 3, \mathrm{C} 3$ & 8 \\
\hline Galanthus nivalis & $\S 3, \mathrm{C} 3$ & 7 \\
\hline Equisetum ramosissimum & $\S 3, \mathrm{C} 3$ & 1 \\
\hline Pulicaria dysenterica & $\mathrm{C} 1$ & 12 \\
\hline Ranunculus sardous & $\mathrm{C} 1$ & 2 \\
\hline Carex strigosa & $\mathrm{C} 2$ & 241 \\
\hline Cardamine dentata & $\mathrm{C} 2$ & 123 \\
\hline Odontites verna & $\mathrm{C} 2$ & 89 \\
\hline Leonurus marrubiastrum & $\mathrm{C} 2$ & 64 \\
\hline Carex divulsa & $\mathrm{C} 2$ & 47 \\
\hline
\end{tabular}




\begin{tabular}{|c|c|c|}
\hline Species & $\begin{array}{c}\text { threat } \\
\text { category }\end{array}$ & $\begin{array}{l}\text { number of } \\
\text { segments }\end{array}$ \\
\hline Bromus commutatus & $\mathrm{C} 2$ & 19 \\
\hline Sium latifolium & $\mathrm{C} 2$ & 12 \\
\hline Althaea officinalis & $\mathrm{C} 2$ & 6 \\
\hline Cerastium dubium & $\mathrm{C} 2$ & 6 \\
\hline Potamogeton nodosus & $\mathrm{C} 2$ & 5 \\
\hline Hydrocharis morsus-ranae & $\mathrm{C} 2$ & 4 \\
\hline Lycopus exaltatus & $\mathrm{C} 2$ & 4 \\
\hline Sonchus palustris & $\mathrm{C} 2$ & 3 \\
\hline Cnidium dubium & $\mathrm{C} 2$ & 3 \\
\hline Dipsacus laciniatus & $\mathrm{C} 2$ & 2 \\
\hline Centaurium pulchellum & $\mathrm{C} 2$ & 1 \\
\hline Lythrum virgatum & $\mathrm{C} 2$ & 1 \\
\hline Najas marina & $\mathrm{C} 2$ & 1 \\
\hline Parietaria officinalis & $\mathrm{C} 2$ & 1 \\
\hline Lotus tenuis & $\mathrm{C} 3$ & 47 \\
\hline Silaum silaus & C3 & 36 \\
\hline Pseudolysimachion longifolium & C3 & 33 \\
\hline Trifolium fragiferum & $\mathrm{C} 3$ & 22 \\
\hline Verbascum blattaria & $\mathrm{C} 3$ & 16 \\
\hline Butomus umbellatus & $\mathrm{C} 3$ & 13 \\
\hline Virga pilosa & $\mathrm{C} 3$ & 12 \\
\hline Verbena officinalis & $\mathrm{C} 3$ & 9 \\
\hline Corydalis pumila & $\mathrm{C} 3$ & 8 \\
\hline Leersia oryzoides & $\mathrm{C} 3$ & 5 \\
\hline Scrophularia umbrosa & $\mathrm{C} 3$ & 5 \\
\hline Cardamine matthioli & $\mathrm{C} 3$ & 4 \\
\hline Carex curvata & $\mathrm{C} 3$ & 2 \\
\hline Lathyrus latifolius & $\mathrm{C} 3$ & 2 \\
\hline Agrimonia procera & C3 & 1 \\
\hline Cyperus fuscus & $\mathrm{C} 3$ & 1 \\
\hline Lactuca quercina & $\mathrm{C} 3$ & 1 \\
\hline Myosurus minimus & C3 & 1 \\
\hline Potamogeton lucens & $\mathrm{C} 3$ & 1 \\
\hline Thalictrum lucidum & $\mathrm{C} 3$ & 1 \\
\hline Carex riparia & $\mathrm{C} 4 \mathrm{a}$ & 626 \\
\hline Senecio erraticus & $\mathrm{C} 4 \mathrm{a}$ & 154 \\
\hline Aethusa cynapioides & $\mathrm{C} 4 \mathrm{a}$ & 100 \\
\hline Cerastium lucorum & $\mathrm{C} 4 \mathrm{a}$ & 69 \\
\hline Cucubalus baccifer & $\mathrm{C} 4 \mathrm{a}$ & 65 \\
\hline Carex buekii & $\mathrm{C} 4 \mathrm{a}$ & 59 \\
\hline Epipactis helleborine & $\mathrm{C} 4 \mathrm{a}$ & 27 \\
\hline Veronica montana & $\mathrm{C} 4 \mathrm{a}$ & 27 \\
\hline Arum cylindraceum & $\mathrm{C} 4 \mathrm{a}$ & 17 \\
\hline Vicia dumetorum & $\mathrm{C} 4 \mathrm{a}$ & 13 \\
\hline Veronica scutellata & $\mathrm{C} 4 \mathrm{a}$ & 10 \\
\hline Verbascum austriacum & $\mathrm{C} 4 \mathrm{a}$ & 9 \\
\hline Carex disticha & $\mathrm{C} 4 \mathrm{a}$ & 8 \\
\hline
\end{tabular}




\begin{tabular}{|l|c|c|}
\hline \multicolumn{1}{|c|}{ Species } & $\begin{array}{c}\text { threat } \\
\text { category }\end{array}$ & $\begin{array}{c}\text { number of } \\
\text { segments }\end{array}$ \\
\hline Serratula tinctoria & $\mathrm{C} 4 \mathrm{a}$ & 6 \\
\hline Galium boreale & $\mathrm{C} 4 \mathrm{a}$ & 5 \\
\hline Myosotis sparsiflora & $\mathrm{C} 4 \mathrm{a}$ & 5 \\
\hline Barbarea stricta & $\mathrm{C} 4 \mathrm{a}$ & 4 \\
\hline Melica transsilvanica & $\mathrm{C} 4 \mathrm{a}$ & 4 \\
\hline Bromus japonicus & $\mathrm{C} 4 \mathrm{a}$ & 3 \\
\hline Carex otrubae & $\mathrm{C} 4 \mathrm{a}$ & 3 \\
\hline Carex pseudocyperus & $\mathrm{C} 4 \mathrm{a}$ & 3 \\
\hline Centaurium erythraea & $\mathrm{C} 4 \mathrm{a}$ & 3 \\
\hline Lemna trisulca & $\mathrm{C} 4 \mathrm{a}$ & 3 \\
\hline Schoenoplectus lacustris & $\mathrm{C} 4 \mathrm{a}$ & 3 \\
\hline Inula salicina & $\mathrm{C} 4 \mathrm{a}$ & 2 \\
\hline Atriplex oblongifolia & $\mathrm{C} 4 \mathrm{a}$ & 1 \\
\hline Corydalis intermedia & $\mathrm{C} 4 \mathrm{a}$ & 1 \\
\hline Cynodon dactylon & $\mathrm{C} 4 \mathrm{a}$ & 1 \\
\hline Elytrigia intermedia & $\mathrm{C} 4 \mathrm{a}$ & 1 \\
\hline Kohlrauschia prolifera & $\mathrm{C} 4 \mathrm{a}$ & 1 \\
\hline Lavatera thuringiaca & $\mathrm{C} 4 \mathrm{a}$ & 1 \\
\hline Potentilla arenaria & $\mathrm{C} 4 \mathrm{a}$ & 1 \\
\hline Veronica verna & $\mathrm{C} 4 \mathrm{a}$ & 1 \\
\hline Carex chabertii & $\mathrm{C} 4 \mathrm{~b}$ & 21 \\
\hline Xanthium albinum & $\mathrm{C} 4 \mathrm{~b}$ & 6 \\
\hline Cerastium pumilum & $\mathrm{C} 4 \mathrm{~b}$ & 2 \\
\hline
\end{tabular}

\section{DISCUSSION}

Professional literature seldom provides results of a full-area inventory of floodplain forests. Trinajstič et al. (2005) present the richness of flora in floodplain forests of northern Croatia with a number of 437 species. Although our study area lies in the northern part of the Pannonian Basin, the total number of found species is higher than in its southern part, i.e. the species diversity of lowland floodplain forests of northern Croatia (612 versus 437 species of vascular plants). Other data come from geographically distant areas: Lyon and Sagers (1998) found 65 families in the floodplain of the Current and Jacks Fork Rivers, North America, and in agreement with our results they ascertained low fidelity of the found species - only fidelity of 42 species out of the 269 recorded ones was over $10 \%$. Tabacchi et al. (1996) found 1,396 plant species along the entire corridor of the Adour River (SW France), which accounts for a fifth of the flora of France. The species diversity of southMoravian floodplain forests we established also corresponds to about a fifth of the flora of the Czech Republic. The highest biodiversity was found by Pott et al. (2011) in the tropical South American Pantanal wetland, where nearly 2,000 species were recorded in an area of over $150,000 \mathrm{~km}^{2}$ in various types of vegetation. The study of Schnitzler et al. (2007) summarised available articles focused on the diversity of riparian forests across the whole of Europe and recorded 1,380 species. Even these sparse data testify to the considerable significance of floodplain forests for the maintenance of diversity of vascular plants.

Most authors examine the species diversity of floodplain forests using sample plots, not full-area terrain surveys, and thus they naturally reach lower numbers than we have 
recorded. Ernault et al. (2006) found 334 species of plants in twenty plots of the Seine floodplain forests, each having an area of $1 \mathrm{~km}^{2}$. Goebel et al. (2006) used 417 plots of $1 \mathrm{~m}^{2}$ in size and determined 162 species of plants in various river systems in NE Wisconsin. Mölder et al. (2011) explored the diversity of flora along the Danube River and found 165 species of higher plants, including 22 graminoids (13\%), 100 forbs (61\%) and 43 woody species $(26 \%)$. Only woody plants were examined by Santos (2010). She recorded 53 species, out of which 28 were endemic, in 70 river plots of $2 \mathrm{~km}$ in length on the Sado and Guadiana watersheds in southern Portugal. Paal et al. (2007) studied the floodplain forests in Estonia in 79 subnatural stands. The ground vegetation was described using randomly located sample quadrats of 1 x $1 \mathrm{~m}$; their number was $15-20$ per stand. The total species list included 372 plant species: 17 species in the tree layer, 17 in the shrub layer, 225 in the herb layer and 100 species in the moss layer. A total of 269 herb and 70 tree species were identified on 94 sample plots by Lyon and Sagers (1998), within the Ozark National Scenic Riverways (ONSR), a forest corridor enclosing a $161 \mathrm{~km}$ stretch of the Current River and a $55 \mathrm{~km}$ stretch of the Jacks Fork River in southwest Missouri, USA. Similarly, McLane et al. (2012) recorded 193 plant species within the Cypress Creek NWR, Illinois, U.S.A., out of which 56 were woody plants, in 80 plots of $1 \mathrm{~m}^{2}$. Archaux et al (2010) sampled vascular flora in 181 poplar plantations along the Seine and Aube rivers within plots of $200 \mathrm{~m}^{2}$ in area. They recorded 211 plant species (32 forest species, 40 tall herbs and 48 meadow plants, 38 ruderal species, 53 other species - aquatic, generalist, field plants). Schnitzler (1997) showed 106-157 plant species (incl. 37-56 woody species) from the Ill, Rhine, Loire and Allier floodplains, Trémolieres et al. (1998) presented only 37 woody species in the Rhine floodplain, but these were bound to the communities of alluvial hardwood forests. Godreau et. al. (1999) in their study from the Saone floodplain mentioned 104 plant species in riverine wetlands, 208 plant species in grasslands, unfortunately the number of forest species is not given.

High native plant diversity in riparian biotopes is largely associated with natural disturbance, particularly flooding and scour by seasonal and storm related flood pulses, which create regeneration microsites and mediate resource competition among species (Naiman \& Decamps 1997; Naiman et al. 1993, 2005). Frequent natural or anthropogenic disturbances, however, can also create conditions conducive to alien plant establishment (De Ferrari \& Naiman 1994; Pyšek \& Prach 1994; Planty-Tabacchi et al. 1996; Pyle 1995; Stohlgren et al. 1998).

We found 170 adventive species in the study area, it is $26 \%$ of all vascular plants creating the floodplain forest communities. Williams (2010) described forty alien plant species (17.8\% of the total surveyed flora) from 42 survey sites across the seven islands of the Allegheny River Islands Wilderness (northwestern Pennsylvania). Košir et al. (2013) presented up to $15 \%$ proportion of neophytes in phytocoenological plots along the Mura River (NE Slovenia). Schnitzler et al. (2007) summarised 1,380 species across European riparian forests, $45(3.3 \%)$ of these were exotic species. Many exotics found in their study were introduced intentionally either from North America (51\%) or Asia (38\%). The exotics belong to various life-forms: approximately 50\% are grasses (polycarpic perennials, summer and autumn annuals), while the rest are phanerophytes, equally distributed among trees, shrubs and liana life-forms. Most of the exotics are thermophilous and lightdemanding pioneer species from warm temperate floodplains. Thirty-two percent are from the Asteraceae family. The distribution of exotics in the 177 communities recorded is highly unequal. Twenty-six are present at low levels in very few communities; seven have an intermediate distribution; and twelve (27\%) are abundant in a large range of habitats (in compliance with our results there are for example Impatiens parviflora, Erigeron 
canadensis or Solidago gigantea). McLane et al. (2012) recorded a $14.4 \%$ proportion of exotic species in the basin of the Cypress Creek NWR, Illinois, U.S.A., and Uowolo et al. (2005) even higher $-30 \%$ proportion of exotic species - along the Yampa and Green rivers (northwest Colorado, USA). Concerning neophytes, the Upper Danube flora yielded five species (7\%), the Middle Danube Flora nine species (14\%) and the Lower Danube Flora eight species (10\%). The most important non-native tree species were Fraxinus pennsylvanica (Middle and Lower Danube), Acer negundo (Middle Danube) and Robinia pseudoacacia (Upper Danube), which reached considerable proportions in the tree layer. Frequent non-native herb species were Impatiens parviflora and Solidago gigantea (Upper and Middle Danube), Aster parviflorus, Oxalis stricta (Middle Danube) and Aster lanceolatus (Lower Danube). The invasive shrub species Amorpha fruticosa was very common on the Lower Danube (Mölder \& Schneider 2011). Also Chmura \& Sierka (2006) in their study of Polish floodplain forests consider Impatiens parviflora to be a significant invasive species. Magee et al. (2008) evaluated the importance of alien species in the existing vegetation along wadeable streams of a large, topographically diverse river basin in eastern Oregon, USA; they identified 60 alien species and 355 native species. Alien species occurred in $93 \%$ of sample plots, in all community types, and along all sampled stream reaches, with relative alien cover (RAC) ranging from $0.1 \%$ to $47 \%$ and 1 to 24 alien species occurring along individual stream reaches. RAC differed among community types: it was the greatest in arid associations (shrubland/grassland), followed by associations with limited tree canopy cover (meadows, dry forest), and the lowest in moist, closed forest associations.

Floodplains are considered vulnerable to exotic species (Hood \& Naiman 2000; Harris et al. 2005), due to the combined influence of intensive human exploitation, a high degree of hydrological connectivity that facilitates propagule dispersal and the high spatial and temporal heterogeneity inherent to these systems. Globally, anthropogenic alterations to floodplain hydrological regimes have frequently resulted in riparian species invasions (Richardson et al. 2007). Vegetation changes are partially structured by reduced flood frequency favouring increased abundance of exotic, sexually reproducing annuals at drier sites. Sites of low flood frequency are more sensitive to future exotic weed invasion. Flow restoration is predicted to benefit propagule dispersal of species adopting dual regeneration strategies, which are predominantly natives in this system (Stokes et al. 2010). The invasion by alien plant species is a major challenge to the conservation and management of riparian areas, which can alter ecosystem structure and function in undesirable ways (Hood \& Naiman 2000; Stohlgren et al. 1998). The invasive species capable of becoming dominant are the most dangerous, and in the study area it is Aster lanceolatus (Řepka et al. 2009). Brewer (2010) described a similar example: a significant negative effect of species richness on invasive grass $M$. vimineum abundance. Altogether, his results suggest that the same factors that reduce biotic resistance have even greater direct positive effects on the abundance of invasive grass and native floodplain specialists. According to investigation of Saccone et al. (2010), Acer negundo showed both a high survival in the shade and a high growth in full light. This species could be an example of adaptive plasticity that certainly represents a competitive advantage over native species. Another example is mentioned by Hanula \& Horn (2011); they investigated the effects of the invasive shrub Chinese privet (Ligustrum sinense) and two methods (mulching or hand-felling) of removing it from riparian forests on butterfly communities. Pyšek \& Prach (1993) named four significant invasive species in riparian habitats of central Europe: Impatients glandulifera, Heracleum mantegazzianum, Reynoutria japonica and R. sachalinensis; none of these has caused a significant problem in the study area. 
Technical regulations of the water regime within floodplains can also impact on the species diversity of floodplain forests. For example, Trémoliéres et al. (1998) compared various sections of an alluvial hardwood forest along the Rhine. Using six plots of about $2,000 \mathrm{~m}^{2}$, they found 63 species (25 woody species) in a flooded floodplain, 121 species (45 woody species) in a floodplain that had not been flooded for 30 years, and 95 species (47 woody species) in a floodplain not flooded for 130 years. Deiller et al. (2001) mentioned that the species richness of the extant vegetation increases with the duration of interruption of the floods in the Rhine forest as a result of introduction of flood-intolerant species in the unflooded forest. By contrast, Uowolo et al. (2005) recorded a $40 \%$ higher number of species in unregulated floodplain of the Yampa river in contrast to the regulated Green River. Other authors also document the changes in species composition and spatial structure of the synusia of floodplain forest herb layer (Vašíček 1985, Vrška 1997, 1998, Maděra 2001a, 2001b; Viewegh 2002, Unar \& Šamonil 2008, Santos 2010) or in the tree layer (Schnitzler 1994; Trémoliéres et al. 1998; Janík et al. 2008, 2011) in dependence on drying of floodplain forests, when flood-intolerant and mesic species can arrive.

Much fewer studies deal with threatened species in floodplains. De Nooij (2006) presented 136 threatened species of vascular plants related to occurrence in river floodplains in Holland. Godreau et al. (1999) found 31 regionally threatened plant species in all biotopes (wetlands, grasslands) in the Saone floodplain; surprisingly, in floodplain forests none such species was found. Similarly, a study by Ot'ahelová et al. $(1992,1997)$ mentioned 129 rare or threatened plant and moss species in the Slovakian part of the Morava river polder. Based on their results, the biotope of floodplain forest is insignificant for the threatened species, comprising only $4 \%$ of found species. Floodplain meadows, water biotopes, wetlands, acid sands, bare bottoms and even anthropogenic biotopes (dams, road edges, fallows) are more significant as they contain more threatened species. However, our results show that the floodplain forests are significant biotopes for threatened species we have recorded a total of 113 , which is $19.46 \%$ of all species. This discrepancy can be perhaps explained by the insufficiently consistent approach of the florists to the full-area forest inventory as the orientation in them is difficult and demanding. A lot of species occur in forests only rarely and they need not be discovered unless the forest is scoured thoroughly.

\section{CONCLUSIONS}

From the perspective of species diversity of vascular plants, floodplain forests in the Tvrdonice forest district are a highly valuable area containing many threatened species. However, there is a high proportion of adventive species. The most significant of them is Aster lanceolatus, which poses a serious problem due to its presence in nearly $95 \%$ of segments as well as its ability to create dominant stands considerably reducing the species diversity.

The high number of adventive species and their relatively high occurrence are caused by a number of factors. The most significant of them are the used ways of stand regeneration with the broadcast soil preparation - agroforestry in history and in more recent decades ploughing, raking of the soil profile with stumps into mounds, or milling (Řepka, Maderra 2009b). The primary vector for the spread of these species is the river and its flooding system; however, stand fragmentation (Dynesius \& Nilsson 1994) and their connection by forest roads, clearings, and canals also played their role after the regulation of the Morava river and elimination of regular floods (Penka et al. 1991, Horák 1964). 
The full-area floristic inventory provides unique results. It expands our knowledge about the significance of floodplain forests for biodiversity and enables us to create maps of occurrence of particular species, places with high diversity and places with the troublesome occurrence of adventive species. These geographic models can be well used for the management of forest stands or the zonation of Dolní Morava Biosphere Reserve.

\section{ACKNOWLEDGEMENT}

The results could be attained thanks to support provided by NAZV (National Agency for Research in Agriculture) project called Harmonization of Forest Management in Floodplains as a Tool to Preserve Species Diversity of Vascular Plants (reg. no. QI92A031).

\section{REFERENCES}

Archaux, F., Chevalier, R., Berthelot, A., (2010). Towards practices favourable to plant diversity in hybrid poplar plantations. Forest Ecology and Management 259: pp.2410-2417

Boedeltje, G., Bakker, J.P., Brinke, A.T., Van Groenendael, J.M., Soesbergen, M., (2004). Dispersal phenology of hydrochorous plants in relation to discharge, seed release time and buoyancy of seeds: The flood pulse concept supported. Journal of Ecology, 92 (5): pp. 786796.

Bohn, U., Neuhäusl, R., Gollub, G., Hettwer, C., Neuhäuslová, Z., Schlüter, H., Weber, H., (2003). Map of the Natural Vegetation of Europe. Scale 1:2 500 000. Federal Agency for Nature Conservation, Bonn, 655 p.

Brewer, J.S. (2010). A Potential Conflict between Preserving Regional Plant Diversity and Biotic Resistance to an Invasive Grass, Microstegium vimineum. Natural Areas Journal, 30(3): pp. 279-293.

Chmura, D., Sierka, E., (2006).Relation between invasive plant and species richness of forest floor vegetation: A study of Impatiens parviflora DC. Polish Journal of Ecology, 54 (3): pp. 417-428

Danihelka, J., Grulich, V., Šumberová, K., Řepka, R., Husák, Š. et Čáp, J., (1995). O rozšíření některých cévnatých rostlin na nejjižnější Moravě. Zprávy České Botanické Společnosti, 30 (Suppl.): pp. 29-102.

Danihelka, J., Šumberová, K. (2004). O rozšǐření některých cévnatých rostlin na nejjižnější Moravě II. Př́roda, Praha, 21: pp.117-192.

De Ferrari, C. M., Naiman, R. J., (1994). A multi-scale assessment of the occurrence of exotic plants on the Olympic Peninsula, Washington. Journal of Vegetation Science 5: pp. 247-258.

Deiller, A.F., Walter, J.M.N., Trémoliéres, M., (2001). Effect of flood interruption on species richness, diversity and floristic composition of woody regeneration in the upper Rhine alluvial hardwood forest. Regulated Rivers: Research \& Management, 17: pp. 393405

De Nooij, R.J.W., Lotterman, K.M., van de Sande, P.H.J., Pelsma, T., Leuven, R.S.E.W., Lenders, H.J.R., (2006). Validity and sensitivity of a model for assessment of impacts of 
river floodplain reconstruction on protected and endangered species. Environmental Impact Assessment Review 26: pp. 677-695

Dynesius, M., Nilsson, C., (1994). Fragmentation and flow regulation of river systems in the northern third of the world. Science 266: pp. 753-761.

Ernoult, A., Tremauville, Y., Cellier, D., Margerie, P., Langlois, E., Alard, D., (2006). Potential landscape drivers of biodiversity components in a flood plain: Past or present patterns? Biological Conservation, 127: pp. 1 -1 7

Godreau, V., Bornette, G., Frochot, B., Amoros, C., Castella, E., Oertli, B., Chambaud, F., Oberti, D., Craney, E., (1999). Biodiversity in the floodplain of Saone: a global approach. Biodiversity and Conservation, 8: pp. 839-864.

Goebel, P.CH., Pregitzer, K.S., Palik, B.J., (2006). Landscape hierarchies influence riparian ground-flora communities in Wisconsin, USA. Forest Ecology and Management 230: pp. 43-54

Hanula, J.L., Horn, S., (2011). Removing an exotic shrub from riparian forests increases butterfly abundance and diversity. Forest Ecology and Management 262: pp. 674-680.

Harris, M.B., Tomas, W., Mourao, G., Da Silva, C.J., Guimaraes, E., Sonoda, F., Fachim, E., (2005). Safeguarding the Patanal wetlands: threats and conservation initiatives. Conservation Biology 19: pp. 714-720

Holub, J., Procházka, F., (2000). Red List of vascular plants of the Czech Republic. Preslia, 72: pp. 187-230.

Hood, G.W., Naiman, R.J., (2000). Vulnerability of riparian zones to invasion by exotic vascular plant species. Plant Ecology, 148: pp. 105-114

Horák, J., (1961). Jihomoravské lužní lesy (typologická studie). Thesis, VŠZ, Brno, 266 pp. Horák, J., (1964). Lesní fytocenóza jako indikátor změn vodního režimu lužních lesů. In: Vegetační problémy budování vodnich děl, (pp. 39-53).ČSAV Praha

Hrib, M., (2004). Z historie lesního hospodářství. In. Hrib, M., Kordiovský, E. (eds.): Lužní les v Dyjsko-moravské nivě. (pp. 209-226). Moraviapress, Břeclav.

Janík, D., Adam, D., Vrška, T., Hort, L., Unar, P., Král, K., Šamonil, P., Horal, D., (2008). Tree layer dynamics of the Cahnov-Soutok near-natural floodplain forest after 33 years (1973-2006). European Journal of Forest Research, 127 (4): pp. 337-345.

Janík, D., Adam, D., Vrška, T., Hort, L., Unar, P., Král, K., Šamonil, P., Horal, D., (2011). Field maple and hornbeam populations along a 4-m elevation gradient in an alluvial forest. European Journal of Forest Research, 130: pp. 197-208.

Klimo, E., Hager, H., (2000). The floodplain forests in Europe. EFI, Leiden, Boston, Köln, Brill, 215 p.

Klimo, E., Hager, H., Matić, S., Anić, I., Kulhavý, J., /eds./ (2008). Floodplain Forests of the Temperate Zone of Europe. Lesnická práce, Kostelec nad Černými Lesy, 623 p.

Košir, P., Čarni, A., Marinšek, A., Šilc, U., (2013). Floodplain forest communities along the Mura River (NE Slovenia). Acta Botanica Croatia, 72, 1: pp. 71-95.

Kubát, K., Hrouda, L., Chrtek, J., Kaplan, Z., Kirschner, J., Štěpánek, J., (2002). Klíč ke květeně České republiky. Academia, Praha, 928 p.

Lyon, J., Sagers, C.L., (1998). Structure of herbaceous plant assemblages in a forested riparian landscape. Plant Ecology 138: pp. 1-16. 
Nožička, J., (1956). Z minulosti jihomoravských luhů (Předběžná studie). Práce výzkumných ústavi̊ lesnických ČSR, sv.10: pp. 169-199.

Maarel van der, E., (1975). Man-made natural ecosystems in environmental management and planning. In: Dobben van, W.H., Lowe-McConell, R. H., (eds.): Unifying concepts in ecology. (pp. 263 - 274).The Hague, Dr W. Junk B. V. Publishers.

Maděra, P., (2001a). Response of floodplain forest communities herb layer to changes in the water regime. Biológia, Bratislava, 56: pp. 63-72.

Maděra, P., (2001b). Effect of water regime changes on the diversity of plant communities in floodplain forests. Ekológia (Bratislava), Vol.20, Supplement 1, pp.116-129.

Maděra, P., et al. (2007). 100 nejzajímavějšich stromů Biosférické rezervace Dolní Morava. BR Dolní Morava, 120 p.

Maděra, P., Vukelic, J., Buček, A., Baričevic, D., (2008). Floodplain forest plant communities. In. Klimo, E. et al., (eds.): Floodplain forests of the temperate zone of Europe. (pp. 102-159). Lesnická práce, Kostelec nad Černými lesy.

Maděra, P., Šebesta, J., Řepka, R., Klimánek, M., (2011). Vascular plants distribution as a tool for adaptive forest management of floodplain forests in the Dyje river basin. Journal of Landscape Ecology, 4, 2: pp. 18-34.

Magee, T.K., Ringold, P.L., Bollman, M.A., (2008). Alien species importance in native vegetation along wadeable streams, John Day River basin, Oregon, USA. Plant Ecology 195: pp. 287-307

McLane, C.R., Battaglia, L.L., Gibson, D.J., Groninger, J.W., (2012). Succession of Exotic and Native Species Assemblages within Restored Floodplain Forests: A Test of the Parallel Dynamics Hypothesis. Restoration Ecology, 20, 2: pp. 202-210.

Mölder, A., Schneider, E., (2011). On the beautiful diverse Danube? Danubian floodplain forest vegetation and flora under the influence of river eutrophication. River Research and Applications, 27: pp. 881-894

Naiman, R.J., Decamps, H., Pollock, M., (1993). The role of riparian corridors in maintaining regional biodiversity. Ecological Applications, 3: pp. 209-212

Naiman, R. J., Decamps, H., (1997). The ecology of interfaces: Riparian zones. Annual Review of Ecology, Evolution and Systematics, 28: pp. 621-658.

Naiman, R. J., Decamps, H., MC Clain, M.E., (2005). Riparia: Ecology, Conservation, and Management of Streamside Communities. Elsevier Academic Press, New York.

Neuhäuslová, Z., (2001). L2 Lužní lesy. In: Chytrý, M., Kučera, T., Kočí, M. (eds.): Katalog biotopů České republiky, (pp. 173-179). AOPK, Praha.

Ot’ahelová, H., Banásová, V., Jarolímek, I., Husák, Š., Zaliberová, M., Zlinská, J., (1992). K výskytu ohrozených druhov flóry Slovenska v inundačnom území dolného toku rieky Moravy. Bulletin Slovenskej Botanickej Spoločnosti, Bratislava 14: pp. 34-35.

Ot’ahelová, H., Banásová, V., Jarolímek, I., Zaliberová, M., (1997). Zoznam ohrozených druhov rastlín v nivě Moravy. Bulletin Slovenskej Botanickej Spoločnosti, Bratislava 19: pp. 107-113.

Paal, J., Rannik, R., Jeletsky, E.M., Prieditis, N., (2007). Floodplain forests in Estonia: Typological diversity and growth conditions. Folia Geobotanica 42: pp. 383-400.

Penka, M., Vyskot, M., Klimo, E., Vašíček, F., (1985). Floodplain forest ecosystem 1. Academia, Praha, 466 p. 
Penka, M., Vyskot, M., Klimo, E., Vašíček, F., (1991). Floodplain forest ecosystem 2. Academia, Praha, 632 p.

Planty-Tabacchi, A., Tabacchi, E., Naiman, R. J., De Ferrari, C. M., Decamps, H., (1996). Invasibility of species-rich communities in riparian zones. Conservation Biology, 10: pp. 598-607.

Pott, A., Oliveira, A.K.M., Damasceno-Junior, G.A., Silva, J.S.V., (2011). Plant diversity of the Pantanal wetland. Brazilian Journal of Biology, 71, 1 (suppl.): pp. 265-273.

Pyle, L. L., (1995). Effects of disturbance on herbaceous exotic plant species on the floodplain of the Potomac River. American Midland Naturalist, 134: pp. 244-253.

Pyšek, P., Prach, K., (1993). Plant invasions and the role of riparian habitats: a comparison of four species alien to central Europe. Journal of Biology, 20: pp. 413-420.

Pyšek, P., Prach, K., (1994). How Important are Rivers for Supporting Plant Invasions? In. L. C. de Waal, L. E. Child, P. M. Wade and J. H. Brock (Eds.): Ecology and Management of Invasive Riverside Plants. (pp. 19-26). John Wiley \& Sons Ltd.

Pyšek, P., Sádlo, J., Mandák, B., (2002). Catalogue of alien plants of the Czech Republic. Preslia, 74: pp. 97-186.

Richardson, D., Holmes, P.M., Elser, K.J., Galatowitsch, S.M., Stromberg, J.C., Kirkman, S.P., Pyšek, P., Hobbs, R.J., (2007). Riparian vegetation: degradation, alien plant invasions, and restoration prospects. Diversity and Distributions, 13: pp. 126-139

Rychtecká, P., Dreslerová, J., (2009). Important woody species in Poodří floodplains (Czech Republic). Journal of Landscape Ecology, 2(1): pp. 58-76.

Řepka, R., Maděra, P., (2009). Rozšíření adventivních druhů v nížinných luzích jižní Moravy - případ hvězdnice kopinaté (Aster lanceolatus). In. Měkotová, J., (ed.): Řični krajina 6. (pp. 100-106). Sborník příspěvků z konference 21.ř́ijna 2009, Olomouc.

Řepka, R., Maděra, P., (2009b). Diverzita vyšších cévnatých rostlin lužního lesa ve vztahu k jeho věku. Zprávy České Botanické Společnosti, 44, Materiály 24: pp. 101-110.

Saccone, P., Brun, J.J., Michalet, R., (2010). Challenging growth-survival trade-off: A key for Acer negundo invasion in European floodplains? Canadian Journal of Forest Research, 40, 10: pp. 1879-1886.

Santos, M.J., (2010). Encroachment of upland Mediterranean plant species in riparian ecosystems of southern Portugal. Biodiversity and Conservation, 19: pp.2667-2684.

Schnitzler, A., (1994). Conservation of biodiversity in alluvial hardwood forests of the temperate zone. The example of Rhine valley. Forest ecology and management, 68: pp. 385-398.

Schnitzler, A., (1997). River dynamics as a forest process: interaction between fluvial systems and alluvial forests in large European river plains. The botanical review, 63, 1: pp. 40-64.

Schnitzler, A., Hale, B.W., Alsum, E.M., (2007). Examining native and exotic species diversity in European riparian forests. Biological Conservation, 138: pp. 146-156.

Stohlgren, T. J., Bull, K. A., Otsuki, Y., Villa, C. A., Lee, M., (1998). Riparian zones are havens for exotic plant species in the central grasslands. Plant Ecology, 138: pp. 113-125.

Stokes, K., Ward, K., Colloff, M., (2010). Alterations in flood frequency increase exotic and native species richness of understorey vegetation in a temperate floodplain eucalypt forest. Plant Ecology, 211: pp. 219-233. 
Tabacchi, E., Planty-Tabacchi, A.M., Salinas, M.J., Decamps, H., (1996). Landscape structure and diversity in riparian plant communities: a longitudinal comparative study. Regulated Rivers: Research \& Management, 12: pp. 367-390.

Tremolieres, M., Sanchez-Perez, J.M., Schnitzler, A., Schmitt, D., (1998). Impact of river management history on the community structure, species composition and nutrient status in the Rhine alluvial hardwood forest. Plant Ecology 135: pp. 59-78.

Trinajstič, I., Franjič, J., Škvorc, Ž., (2005). The flora of floodplain and marshy forests. In: Vukelič, J., (ed.): Floodplain Forests in Croatia, (p. 93-101), Academy of Forestry Sciences, Zagreb.

Unar, P., Šamonil, P., (2008). The evolution of natural floodplain forests in South Moravia between 1973 and 2005. Journal of Forest Science, 54 (8): pp. 340-354.

Uowolo, A.L., Binkley, D., Adair, E.C., (2005). Plant diversity in riparian forests in northwest Colorado: Effects of time and river regulation. Forest Ecology and Management 218: pp. 107-114.

Úradníček, L., Maděra, P., Tichá, S., Koblížek, J., (2010). Woody Plants of the Czech Republic. Lesnická práce, Kostelec nad Černými lesy, 368 p.

Vašíček, F., (1985). Changes in the herbal vegetation along the topographical moisture gradient. In: Penka, M., Vyskyt, M, Klimo, E., Vašíček, F.: Floodplain Forests Ecosystem 2: (pp, 355-386), Academia, Praha.

Vicherek, J., et al. (2000). Flóra a vegetace na soutoku Moravy a Dyje. Masarykova univerzita v Brně, Brno, 368 p.

Viewegh, J., (2002). South-Moravian floodplain forest herb vegetation in the period 1978 1997. Journal of Forest Science, 48: pp. 88-92.

Vrška, T., (1997). Prales Cahnov po 21 letech (1973-1994). Lesnictví-Forestry 43: pp. 155180.

Vrška, T., (1998). Prales Ranšpurk po 21 letech (1973-1994). Lesnictví-Forestry, 44: pp, 440-473.

Williams, CH.E., (2010). Survey of the alien flora of the Allegheny river island wilderness, Pennsylvania. Rhodora, 112, 950: pp. 142-155

Wenger, E.L., Zinke, A., Gutzweiler, K.A., (1990). Present situation of the European floodplain forests. Forest Ecology and Management, 33-34: pp. 5-12. 